\title{
ICRF HEATING IN TOKAMAKS
}

BY.

H. TAKAHASHI

\section{MASTER \\ PLASMA PHYSICS LABORATORY}

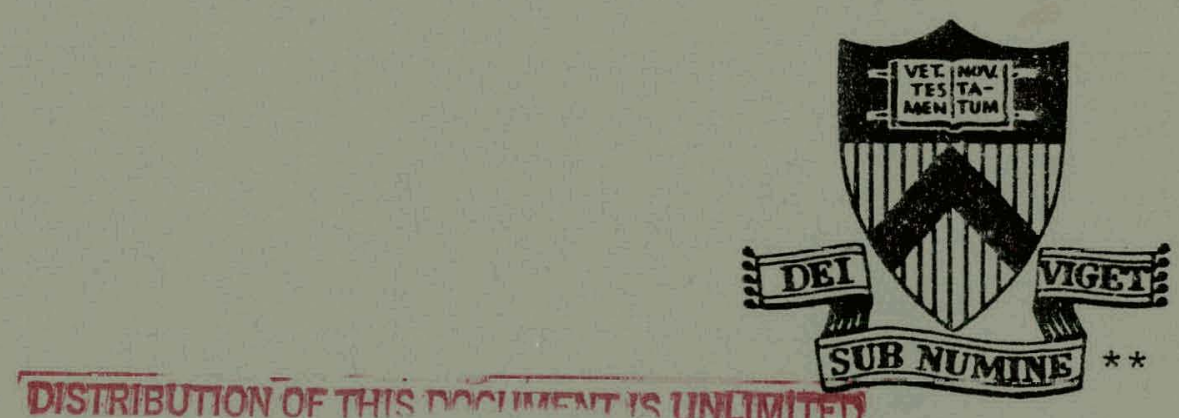

\section{PRINCETON \\ UNIVERSITY PRINCETON, NEW JERSEY}

This work was supported by U. S. Energy Research and Development. Administration Contract $\mathrm{EY}-76-\mathrm{C}-02-3073$. Reproduction, translation, publication, use and disposal, in whole or in part, by or for the United States Government is permitted. 


\section{DISCLAIMER}

This report was prepared as an account of work sponsored by an agency of the United States Government. Neither the United States Government nor any agency Thereof, nor any of their employees, makes any warranty, express or implied, or assumes any legal liability or responsibility for the accuracy, completeness, or usefulness of any information, apparatus, product, or process disclosed, or represents that its use would not infringe privately owned rights. Reference herein to any specific commercial product, process, or service by trade name, trademark, manufacturer, or otherwise does not necessarily constitute or imply its endorsement, recommendation, or favoring by the United States Government or any agency thereof. The views and opinions of authors expressed herein do not necessarily state or reflect those of the United States Government or any agency thereof. 


\section{DISCLAIMER}

Portions of this document may be illegible in electronic image products. Images are produced from the best available original document. 


\section{NOTICE}

This report was prepared as an account of work sponsored by the United States Government. Neither the United States nor the United States Energy Research and Development Administration, nor any of their employees, nor any of their contractors, subcontractors, or their employees, makes any warranty, express

or implied, or assumes any legal liability or responsibility for the accuracy, completeness or usefulness of any information, apparatus, product or process disclosed, or represents that its use would not infringe privately owned rights.

Printed in the United States of Anerica. Available from

National Technical Information Service

U. S. Department of Commerce 5285 Port Royal Road

Springfield, Virginia 22151

Price: Printed Copy \$_*; Microfiche $\$ 3.00$

*

$1-50$

$51-150$

$151-325$

$326-500$

501-1000
NTIS

Selling Price

$\$ 4.00$

5.45

7.60

10.60

13.60 


\section{ICRF HEATING IN TOKAMAKS}

H. Takahashi

Plasma Physics Laboratory, Princeton University Princeton, N. J. 08540 , U. S. A.

This report was prepared as an account of work sponsored by the United States Government. Nelther the United States nor the United Stanes nor any of Research enloyes, nor any of their contractors, subcontractors, or their employees, makes any uniranty, express or implied, or assumes any leza thability or responsibility for the accuracy, completeress os usefulness of any in tormation, apparatu, produ or process discilosed, or redringe privaly owned rights. 
ICRF Heating in Tokamaks

H. Takahashi

Plasma Physics Laboratory, Princeton University Princeton, N. J. 08540 , U. S. A.

\section{ABSTRACT}

The current status of ICRF heating of Tokamak plasmas is described. Two aspects of the subject are discussed: the physics of wave damping and the evaluation of ion heating. Recent experiments in several Tokamak devices at the second harmonic of the deuteron cyclotron frequency have exhibited large discrepancies between the observed and predicted wave damping. Current thinking attributes these discrepancies to the influence of a two-ion hybrid resonance between the majority deuterium ions and impurity protons. A number of experimental observations appear to be consistent with this view. However, the details of the physical processes which lead to wave damping are still unclear. One theory attributes the damping to a linear mode conversion at the twoion hybrid laycr. This proccsa is important, if the proton to deuteron density ratio is large (> 10\%). Another possible explanation, based upon enhanced cyclotron harmonic damping due to the proximity of the two-ion hybrid and cyclotron resonance layers, is discussed in this paper. The boundary value problem for fast wave propagation in an inhomogeneous two-ion plasma has been solved explicitly to obtain the wave field structure. For modest proton concentrations $(<5 \%)$ a steep gradient in the left-hand polarized electric field is found in the region between the hybrid and 
cyclotron resonant layers. This gradient appears to provide sufficient enhancement of the second harmonic damping strength to account for the observed discrepancies. However, a reliable measurement of the proton concentration is needed to choose between the two possibilities. Ion heating was examined in detail in recent experiments in the ATC device. It is shown that ICRF heating can heat the bulk of the ions in the core of a Tokamak plasma with efficiencies ranging from 10 to $40 \%$, without causing any significant change in the ion energy confinement. 


\section{INTRODUCTION}

It is generally believed that ohmic heating in Tokamak devices is not sufficient and therefore some form of additional heating will be required to heat the ions to thermonuclear temperatures. One such method of additional heating currently under intensive experimental and theoretical investigation is irradiation of the plasma by electromagnetic fields in the ion cyclotron range of frequencies ("ICRF" heating). The impressed radio frequency ( $r f$ ) field generates in the plasma fast compressional Alfven waves whose energy is absorbed by the ions (and possibly also by electrons) through various field-particle resonant interactions. Requirements of the ICRF heating for access to the plasma are modest. In this respect, the subject is of particular interest for Tokamaks with limited access for which neutral beam heating may be difficult. While D-T plasmas are of interest from reactor considerations, this paper reviews the current status of ICRF heating of deuterium plasmas near the second harmonic of the deuteron cyclotron frequency.

Usefulness of cyclotron damping as a means of increasing ion energy was successfully demonstrated in linear geometries such as mirror devices and the straight segment of the race-track shaped C-Stellarator [see, e.g., ROTHMAN, et al. (1)]. In these experiments, the slow branch of the low frequency cold plasma wave, also called the torsional Alfven wave or the ion cyclotron wave, was utilized in magnetic beach configurations. Use of the fast branch (the compressional Alfven wave, or the fast wave) at the. 
fundamental and second harmonic of the ion cyclotron frequency $\left(\Omega \equiv \omega / \omega_{c i}=1\right.$ or 2$)$ in toroidal devices was first suggested by ADAM and SAMAIN (2). In toroidal geometry one possible mechanism for dissipating wave energy is again ion cyclotron damping. However, in the inhomogeneous B-field of a Tokamak the cyclotron resonant conditions $(\Omega=1$ or 2$)$ are met only on a roughly cylindrical surface at constant major radius. Ion thermal motion parallel to the $\mathrm{B}$-field makes the resonant region a layer of finite thickness which is referred to as a (ion cyclotron) resonant layer. Waves are generated by passing an rf current through a suitable coil or antenna structure located within the Tokamak vacuum chamber but outside the dense plasma core. Tn the ahsence of strong damping, the waves may propagate around the torus many times and produce interference of their fields. Under proper plasma conditions the interference is constructive and the Tokamak vessel acts as a toroidal resonant cavity. These resonances are often referred to as toroidal eigenmode resonances. Studies of wave generation and propagation were the principal subjerta nf early experiments in TO-1 [IVANOV, KOVAN and LOS' (3)], TM-1-VCh [VDOVIN, et al. (4)] and ST [HOOKE and HOSEA (5); HOSEA and HOOKE (6); ADAM, et al. (7)]. An early theoretical analysis of the toroidal resonances was provided by PERKINS, CHANCE and KINDEL (8). These resonances are important for physical and technological reasons. For example, study of their prnperties allowe dotcrmin ation of the wave damping strength, and high loading resistance at the peaks of resonance helps to hold the antenna voltage down and thus reduce the risk of electrical breakdown. The antenna may be a full-turn loop that encloses the plasma column as in the T-4 
device, or a half-turn arc placed on the inner (high field) side of the plasma torus as in ST or on the outer (low field) side as in ATC. It may have a structural member running in the toroidal direction as in TM-1-VCh. An example of such an antenna, used in the recent ATC experiments, is shown in Fig. I.

Understanding of the wave damping processes is one of the principal concerns of the current ICRF theory and experiment. This is the subject of Part I of this paper. Following historical progression of thought on this subject, we first describe earlier theories on the "second harmonic heating" and "minority heating." Next, the results are described from recent experiments carried out in Tokamaks including TFR [TFR Group $(9,10)]$, TM-1-VCh [VDOVIN, SHAPOTOKOVSKII and RUSANOV (11)], T-4 [IVANOV, KOVAN and SOKOLOV (12); BUZANKTN, et al. (13)] and ATC [GREENOUGH, PAOLONI and TAKAHASHI (14)]. These experiments showed, however, large deviation from predictions of these earlier theories. This led to consideration of the influence of two-ion hybrid resonance between the majority deuteron and minority proton species: At sufficiently high proton concentrations a linear mode conversion process can divert the energy into shorter wavelength modes and thus damp the tast waves. At small proton concentrations the hybrid layer lies spatially close to the cyclotron layer and the rapid variation of the wave E-field polarization near the hybrid resonance layer has Important influence on the second harmonic damping. The latter is a situation that has not, so far, been studied and is discussed in this paper.

Rf power high enough to affect the ion thermal energy significantly was employed in recent experiments in TO-1 [IVANOV, KOVAN 
and SVTSCHEV (15)], ST (7), TM-1-VCh (11), T-4 $(12,13)$ and ATC [TAKAHASHI, et al. $(16,17)]$. One observation common to all of these experiments, made through charge exchange neutral diagnostics, is significant alteration of the ion energy distribution and particularly formation of a high energy tail. The non-Maxwellain distribution of the rf heated ions complicates interpretation of the charge exchange spectra and assessment of ion heating. In the latest ATC experiments (17) the nature of the observed ion heating was studied in detail using several diagnostic techniques. This is the subject of Part II of this paper.

\section{PART I - PHYSICS OF ICRF HEATING}

Earlier Theories

The fast wave considered here has predominantly right-handed (with respect to the B-field) polarization. It is, hnwever, the small left-handed polarized component of the wave E-field in the ion cyclotron resonance layer that causes wave damping and ion hcatiny. The resonance layer thickness is given [ADAM and SAMIN (2)] approximately by $2 \mathrm{R}_{0} \mathrm{v}_{\text {thi }} / \mathrm{v}_{\|}$, where $\mathrm{R}_{0}, \mathrm{v}_{\text {thi }}=\left(2 \mathrm{kT} \mathrm{T}_{i} / \mathrm{m}_{i}\right)$ and $v_{\|}=\omega / k_{\|}$are the Tokamak major radius, ion thermal and parallel wave phase speeds, respectively [Fig. 2(a)]. Figure 2(b) depicts an elementary picture of the cyclotron acceleration of a deuterium ion at its second harmonic cyclotron frequency. Since the wave field varies twice as fast as the cyclotron period, the ion would experience acceleration during one half of its Larmor cycle and deceleration during the other half. In a homogeneous field there is no net gain in particle energy. In an inhomogeneous wave field (i.e., non-zero perpendicular wavelength), however, the 
acceleration and deceleration are in general unequal. On taking a statistical average over an ensemble of particles with their motion randomly phased with respect to the wave field, there is a net transfer of energy from the wave to the particles. The strength of wave damping by this mechanism is therefore dependent on the Larmor radius and the gradient of the left-hand polarized electric field. The second harmonic damping is a weak mechanism under present-day Tokamak conditions, unless the gradient becomes very large. On the other hand, if the ion is a proton, it experiences the electric field at its fundamental cyclotron frequency and it keeps accelerating in a familiar spiraling motion. If there are too many protons, their coherent motion tends to shield out the wave electric: field and the wave damping is consequently weak. When there are only a few percent of protons mixed in a deuterium plasma, damping by this mechanism (commonly called minority species heating) can be much stronger than the second harmonic damping. ADAM and SAMAIN (2) showed that both of these physical processes could be used to heat toroidal plasmas. They have calculated the second harmonic cyclotron damping strength in a one-ion species plasma and the fundamental cyclotron damping (by a minority species) in a two-ion species plasma. [In a later section we point out that the second harmonic damping (by the majority species) in a two-ion species plasma may play an important role in many experimental situations because of enhancement of the harmonic damping due to the two-ion hybrid resonance.] They found, as might be expected from the above physical picture, that the power absorption by second harmonic damping is proportional to $\left|E_{+}\right|^{2}$ and to $1 / 2\left(k_{\perp} \rho_{L}\right)$ where $E_{+}$and $\rho_{L}$ are the complex amplitude of the 
left-handed polarized electric field and ion Larmor radius, respectively. The effect of the field gradient is manifest through $k_{\perp}$. (Later we encounter a situation in which the magnitude of complex $k_{\perp}$ calculated for local plasma properties is not large, but the gradient of $\mathrm{E}_{+}$is very large because of rapidly varying polarization.)

A self-consistent treatment of second harmonic cyclotron damping in an inhomogeneous plasma would require dealing with a differential equation of a high orde. STIX (18) introduces an alternative approach depicted in Fig. 2(a). An ion moving along a B-field line which twists around tize magnetic axis goes through the resonant layer twice each complete revolution. on each passage the ion receives a kick in its perpendicular energy. Between passages the ion loses, due to collisions, phase coherence between its motion and the electric field - a basis for using a quasilinear diffusion coefficient to obtain time evolution of the ion distribution function in spite of the monochromatic nature of the wave field. His results for the heating rates are essentially identical to those of Adam and Samain (but see Ref: 23 of Ref. 18). In a similar fashion, PERKINS (see Ref. 4) finds that the quasilinear diffusion coefficient responsible for the second harmonic damping is artually proportional to the perpendicular gradient of the left-handed electric field.

The fast wave dispersion relation can be cast into the following approximate form:

$$
\mathrm{N}_{\perp}^{2}+(1+\varepsilon \Omega) \mathrm{N}_{\|}^{2}=1
$$

where $\mathrm{N}_{\perp}$ and $\mathrm{N}_{n}$ are the perpendicular and parallel components of 
"Alfven refractive index," $\vec{N}=\overrightarrow{\mathrm{k}} \mathrm{V}_{\mathrm{A}} / \omega$. Here, $\varepsilon$ is unity $(*)$ and $V_{A}$ the Alfven speed. STIX (18) expresses the wave damping strength in terms of the quality factor of a resonant cavity. For the second harmonic and minority heating it is given respectively by:

$$
\begin{aligned}
& Q_{I I}=\frac{2\left(\frac{a}{R_{O}}\right)}{\left(k_{\perp} \rho_{L}\right)^{2} \sqrt{I-\left(\frac{\left.r_{\text {res }}\right)^{2}}{a}\right.}} \\
& Q_{I}=2\left(\frac{a}{R_{O}}\right)\left(\frac{{ }^{n} D}{n_{H}}\right)\left\{1+\frac{9 \pi}{16}\left(\frac{n_{H}}{n_{D}} \frac{v_{I I}}{v_{H}}\right)^{2}\right\}
\end{aligned}
$$

where a and $r_{\text {res }}$ are respectively the plasma minor radius and the distance between the cyclotron resonance layer and the magnetic axis. $\mathrm{n}_{\mathrm{D}}, \mathrm{n}_{\mathrm{H}}$ and $\mathrm{V}_{\mathrm{H}}$ are the deuteron and proton densities and proton thermal speed, respectively. At high densities $v_{\|} / v_{H}$ is not too large and the minority damping is much stronger than the second harmonic damping. However, at low densities the wave phase speed is large and this conclusion no longer holds true. The quality factor is related to the wave damping length through

$$
I_{D}=\frac{I}{k_{i}}=\frac{2 Q v_{g}}{\omega}
$$

where the parallel group velocity is given by

$$
\mathrm{v}_{\mathrm{g}}=\frac{(1+\varepsilon \Omega)}{\left(1-\frac{\varepsilon}{2} \Omega \mathrm{N}_{\mathrm{N}}{ }^{2}\right)} \cdot \mathrm{N}_{\mathrm{n}} \mathrm{v}_{\mathrm{A}}
$$

(*)An approximation in which $\varepsilon$ is zero is sometimes used. This would result in the group velocity which is too sillall by a factor between 3 and 4.5 [see Eq. (5)]. 
Either $Q$ or $L_{D}$ can be measured experimentally under some circumstances as discussed in the next section.

\section{Wave Damping Experiments}

The strength of wave damping can be determined experimentally by taking advantage of the toroidal eigenmode resonances in several ways. The oscillogram in Fig. 3 shows signals ( $r$ envelopes) from maynetic probes placed within the vacuum vessel but outside the limiter radius. Sharp peaks in the envelope pridently correspond to resonances. These peaks often appear in pairs without a significant trough between the two members of the pair (see, e.g., the second and third peaks at 3 and $5 \mathrm{msec}$ in the oscillogram). Such pairs are at present interpreted as "mode splitting." The waves propagating in the opposite toroidal directions are affected by the poloidal magnetic field in different manners (except for the $\mathrm{m}=0$ mode). Consequently, they come into resonance at two different values of the plasma density. The mode splitting, first predicted by PERKINS and CHANCE (1.9) and investiydted in some netail by ADAM and JACQUINOT $(20)$, is believed to be observed in several experiments $(7,10)$, but detailed confirmation has not yet been reported. Occurrence of the mode splitting is of some practical significance. We first note that each member of the pair represents a resonance created by a unidirectionally traveling wave rather than a standing wave composed of two oppositely travcl ing waves. Verification of this fact is an important evidence for the existence of mode splitting. At such a "traveling wave resonance" the parallel wavelength can be determined by a simple phase comparison of signals from probes positioned at some distance apart in the toroidal direction. This is a much simpler procedure than determination 
of the wavelength of a standing wave. Absence of stationary nodal and loop points also means more uniform heating around the torus. If we ascertain or assume that two neighboring peaks represent resonances with two adjacent toroidal eigenvalues within the same family of minor azimuthal and radial eigenmodes and hence the trough between the peaks represents the anti-resonance conditions, then the wave damping length can be determined from the peak-totrough amplitude ratio as follows: Suppose that the antenna carrying unit rf current generates a wave field of amplitude $u$ in the absence of toroidal effects (i.e., waves do not come back to the antenna location). In a toroidal geometry with dissipation, the waves experience a complex phase shict ${ }_{i} \mathrm{~kL}$ each time they go around the torus, where $\mathrm{L}$ is the toroidal circumference. The total field for an $r f$ current of I for the waves propagating around the torus many times i:; obtained by summing an infinite series:

$$
U=u I+u I e^{i k L}+u I e^{2 i k L}+\ldots=\frac{u I}{\left(1-e^{i k L}\right)}
$$

At resonances and anti-resonances $U$ becomes pure real and is given by

$$
\begin{aligned}
& U_{\mathrm{R}}=\frac{\mathrm{uI}_{\mathrm{R}}}{(1-\alpha)} \\
& \mathrm{U}_{\mathrm{A}}=\frac{\mathrm{uI_{A }}}{(I+\alpha)} .
\end{aligned}
$$

where $\alpha=\exp \left(-k_{i} L\right)$ and $I_{R}$ and $I_{A}$ are the rf current at resonance and anti-resonance. The latter. two quantities are experimentally measurable. Here, we have also assumed the plasma conditions vary 
only slightly between these two points and hence the coupling efficiency represented by $u$ can be regarded constant. Then, this quantity, which is in general not directly measurable, can be eliminated by forming the amplitude ratio at a resonance and an adjacent anti-resonance.. Solving for the wave damping length we obtain

$$
\frac{\mathrm{L}_{\mathrm{D}}}{\mathrm{L}}=\ln \frac{1+\gamma}{1-\gamma}
$$

where $\gamma=I_{A} U_{R} / I_{R} U_{A} \cdot$ The advantage of this formalism is that knowledge of density variation and ccupling efficiency is not needed. The normalized damping length $L_{T} / L$ is the number nf times the waves go around the torus before their amplitude e-folds.

The damping length determined (1.4) in this way for different values of toroidal magnetic field is shown in Fig. 4(a). The abscissa is the major radius location of the cyclotron resonance layer. The ciensity, which is not the same for each case due to varying torojdal field, is low enough $\left(\mathrm{n}_{\mathrm{e}}=0.4-0.9 \times 10^{19} / \mathrm{m}^{3}\right)$ for the $m=0$ azimuthal mode to be cut-off. It is also experimentally verified that the prominent resonant peaks are the $\mathrm{m}=+1$ mode [for the fie].d variation of the form exp $\left.i\left(k_{\|} z+m \theta-\omega t\right)\right]$. Nonetheless, very small amplitude modes (e.g., $m=2$, etc.) may affect significantly the measurement of small amplitude at the antiresonance. The values of $L_{D} / L$ qiven here should therefore he interpreted as a lower bound. Another possible source of error in the measurement is the amplitude variation caused by the motion of plasma toward and away from the probes. In order to partially 
account for these effects, the average of the signals from two probes located near the top and bottom of the plasmas is taken. The quality factor of the cavity can also be determined from signals such as ones shown in Fig. 3. These resonant curves are traced out by varying electron density rather than the wave frequency as is customary in $Q$ measurements. It can be shown using the dispersion relation [Eq. (1)] that these two procedures are approximately equivalent and $Q$ can be calculated by

$$
Q \equiv \frac{\omega_{o}}{\Delta \omega} \cong \frac{\omega_{\text {pio }}}{\Delta \omega_{p i}}=\frac{2 n_{e o}}{\Delta n_{e}}
$$

where $\omega_{\text {pio }}$ and $n_{\text {eo }}$ are the ion plasma frequency and electron density at the resonance and $\Delta \omega_{\mathrm{pi}}$ and $\Delta \mathrm{n}_{\mathrm{e}}$ are the differences in these quantities at two half-power points. This method requires measurement of very small variation in electron density, especially under high-Q conditions. The toroidal resonance is also quite sensitive to a change in density profile such as one caused by internal disruptions (9).

Pronounced decrease in the damping length [Fig. 4(a)] as the cyclotron layer moves into the plasma region indicates presence of strong dampirig processes on or near the resonance layer. Under these conditjons $L_{D} / I$ determined from the amplitude ratio method [Eq. (9)] is 4-10, which is consistent with $Q=20-40$ determined from the resonance width method [Eq. (10)]. These values are far smaller than theoretical predictions. The second harmonic damping theory, Eq. (2), predicts $Q \simeq 171) 0$. The minority heating theory, Eq. (3), gives $Q \simeq 1600-3500$, provided the proton concentration is taken to be $3 \%$ and $v_{n} / v_{H}=25-40$. The latter ratio in 
these low density cases is calculated from the straight cylindrical plasma model and corresponds to the toroidal eigennumber of 2-3. Another notable feature of this curve is that the region of the maximum damping is not symmetric with respect to the magnetic axis at $R=87 \mathrm{~cm}$ and is shifted toward the larger major radius side. This is in contrast to prediction of Eq. (2). Both of these features were also observed in $\operatorname{TFR}$ experiments $(9,10)$. Another manifestation of the asymmetry (21) is shown in Fig. 4(b). The abscissa is identical. to that of Fig. 4(a). The ordinate is the output voltage of a bolometer facing the plasma and is proportional to the power loss due to charge exchange and radiation integrated over the period of rf heating pulse. The net difference between discharges with and without the heating pulse is plotted. A sharp peak is located on the larger major radius side of the magnetic axis where the center of the strong damping region in Fig. $4(a)$ is also located. Under these condltions the rf power is evidently more efficiently absorbed by the ions, some of: which become lost through charge exchange and intercepted by the: bolometer.

An additional observation made in the $\operatorname{TFR}$ experiments $(9,10)$ is that in a "pure" hydrogen plasma the wave damping at the second harmonic protion cyclotron frequency is weak and generally in line with theoretical expectations. In TM-1-VCh experiments (11) a controlled amount of hydrogen was adided to a deuterium plasma and broadening of the resonant peaks was observed. Observations described in this and preceding paragraphs appear to be consistent with the notion of a two-ion hybrid resonance between deuterons and protons. The latter may be incidentally present in small 
quantities in deuterium plasmas as an impurity species. Note that the second harmonic deuteron and fundamental proton cyclotron frequencies are identical. In such a case the hybrid resonance layer is located near the cyclotron resonance layer on its high field side. For the experimental conditions considered here protons are the only minority species with a hybrid resonance within the plasma (see a later section for other possibilities). As will be seen below, effects of the additional damping mechanisms due to the hybrid resonance can be strong and asynmetric with respect to the magnetic axis. In a predominantly hydrogen plasma, on the other hand, no hybrid layer exists within the plasma.

Peaks and troughs of the toroidal resonances also appear in variation of the series loading resistance of the antenna defined as the ratio of the net rf power fed into the antenna to its rf current. Signals from directional couplers measuring the incident and reflected powers and from a probe measuring antenna current are fed into an analog circuit to display the resistance on an oscilloscope. Time evolution of the loading resistance obtained in this way for different levels of electron density is shown in Fig. 5 together with the central electron density variations. The ordinate of each of these oscillograms is proportional approximately - there is some instrumental error - to the loading resistance. The resistance contains power loss from the antenna not directly associated with radiation of the waves (circuit loss, wall loss caused by near field, etc.). At higher densities there is also more than one mode excited simultaneously. For these reasons it is difficult to obtain the damping strength reliably from these data. However, a qualitative trend seems clear: As the density 
increases, the resonant peaks broaden and finally at the highest density shown here they become hardly recognizable. Under these conditions the normalized damping length is probably much smaller than unity. In fact, the measured loading resistance is roughly in line with radiation resistance calculated for an infinite cylindrical geometry. (Because of peculiarity of the vacuum vessel shape, presence of a large port and bumper limiters placed near the antenna, modeling of the antenna region of ATC by a cylindrical waveguide is not accurate.). The magnitude of the loading resistance (values measured at troughs) also increases with increasing electron density (14).

Two-Ion Hybrid Resonance

The two-ion hybrid resonance was discovered by BUCHSBAUM (22). He investigated its use for plasma heating taking advantage of efficient dissipation due to collisions among ions with different charge-to-mass ratios (23). Accessibility of this resonance in inhomogeneous cold plasmas was examined by STIX (24). Effects of this resonance in toroidal geometry were observed by ADAM, ALVAREZ de TOLEDO and FELTIN (25). Observation of plasma heating has been reported by TARASENKO, et al. (26) and by BURCHENKO, et al. (27). KLIMA, LONGINOV and STEPANOV (28) examined accessibility of the hybrid resonance in hot Tokamak plasmas. They considered possibilities of electron heating through a series of mode conversion processes and Landau damping of the fast waves. The two-ion hybrid resonance in Tokamak plasmas falls into a class of wave propagation problems in inhomogeneous media described by a wave equation (so-called BUDDEN's equation) with closely located 
resonance and cut-off layers. This class of problems was first investigated by BUDDEN (29) in connection with radio wave propagation in the ionosphere. Early studies of absorption and reflection at an isolated two-ion hybrid resonance were made by STIX (30). SWANSON (31) examined a mode conversion process of the fast. wave perpendicularly incident on the hybrid resonance/ cut-off layer and obtained absorption, reflection and tunnelling coefficients. He found strong damping of the fast waves by this process in reactor-like D-T plasmas.

It has been suggested that the discrepancies observed in the second harmonic heating experiments were indeed caused by the hybrid resonance due to protons incidentally present in deuterium plasmas. JACQUINOT, MCVEY and SCHARER (32) derived a Budden's equation for obliquely incident fast waves and showed that the fast wave damping observed in the TFR experiments could be quantitatively explained in terms of mode conversion damping, provided the minority proton density was about $20 \%$ of that of the deuterons. Most recently PERKINS (33) analyzed extensively the mode conversion processes. He calculated configurations of resonance/ cut-off pairs in Tokamaks for varieties of $D-T$ and $D-H$ plasmas. Treating the most general case of arbitrary angle of incidence he investigated in detail the fate of wave energy diverted to shortwavelength modes and concluded that these modes are heavily damped and therefore cause no reflection of the wave energy from the boundaries. Under these circumstances the Budden's equation adequately describes absorption of the wave energy through the mode conversion process. 
We begin our discussion of the two-ion hybrid resonance by examining the fast wave dispersion relation [Eq. (I) is obtained from the equation below with further approximation] and E-field polarization given by

$$
\begin{aligned}
\mathrm{n}_{\perp}^{2}=\frac{\left(\mathrm{R}-\mathrm{n}_{\|}^{2}\right)\left(\mathrm{L}-\mathrm{n}_{\|}{ }^{2}\right)}{\left(\mathrm{S}-\mathrm{n}_{\|}{ }^{2}\right)}=2\left(\mathrm{R}-\mathrm{n}_{\|}{ }^{2}\right)-\frac{\left(\mathrm{R}-\mathrm{n}_{\|}^{2}\right)^{2}}{\left(\mathrm{~S}-\mathrm{n}_{\|}^{2}\right)}=2 \mathrm{~F}-\frac{\mathrm{F}^{2}}{\mathrm{H}} \\
\rho=\frac{\mathrm{F}_{\mathrm{x}}}{\mathrm{iE} \mathrm{E}}=\frac{\mathrm{n}}{\mathrm{H}}
\end{aligned}
$$

where

$$
\begin{aligned}
& F \equiv R=n_{\|}^{2} \\
& G \equiv L-n_{\|}^{2} \\
& H \equiv S-n_{\|}^{2} .
\end{aligned}
$$

Here $R, I, S$ and $D$ are the cold plasma dielectric tensor elements and are given by [see $\operatorname{STIX}(24), \mathrm{p} .10]$

$$
\begin{aligned}
& R=I-\sum_{j} \frac{\Omega^{2} p j}{\left(1+\varepsilon_{j}{ }^{\prime}{ }^{\prime}\right.} \\
& \mathrm{T}_{1}=1-\sum_{j} \frac{\Omega^{2} \cdot \mathrm{pj}}{\left(1-\varepsilon_{j}{ }^{\Omega_{j}}\right)} \\
& S=\frac{I}{2}(R+L) \\
& D=\frac{1}{2}(R-L)
\end{aligned}
$$

where $\Omega_{\mathrm{pj}}=\omega_{\mathrm{pj}} / \omega$ and $\Omega_{j}=\omega_{j} / \omega$ are respectively the plasma and cyclotron frequencies of the $j-t h$ species divided by the wave 
angular frequency and $\varepsilon_{j}$ is the sign of the charge. The summation $\sum_{j}$ is over all charged species including electrons. Let us designate any charged species which has the fundamental cyclotron resonance layer within the plasma as "resonant species". In the absence of such resonant species, $\mathrm{R}>0, \mathrm{I}<0$ and $\mathrm{S}<0$ in the vicinity of the second harmonic ion cyclotron frequency of the majority species and the fast wave can propagate $\left(n_{\perp}^{2}>0\right)$. However, the resonant species' contributions to $L$ is positive on the high field sides of their respective cyclotron resonances. The contributions are sufficiently large in the immediate vicinities of the cyclotron layers to produce two-ion hybrid resonance and cut-off conditions given respectively by,

$$
\begin{aligned}
& \mathrm{H}=0 \\
& \mathrm{G}=0
\end{aligned}
$$

An example of such a hybrid resonance/cut-off pair for a D-H plasma is shown schematically in Fig. 6 (a) along with variation of the dielectric tensor elements (Fig. $6(\mathrm{~b})$ ) and the square of the perpendicular refractive index (Fig. $6(\mathrm{c})$ ) alonq the central chord. In the shaded areas of the Fig. $6(\mathrm{a}, \mathrm{c})$ the fast wave is evanescent. The evanescent region between the hybrid resonance and cut-off is usually very thin and the waves can tunnel through this region with only partial reflection and absorption. The first laboratory observation of this Budden tunnelling effect was probably made by YOSHIKAWA; ROTHMAN and SINCLAIR(34). In these experiments the ion cyclotron waves were found to tunnel through a magnetic beach region due to Lile presence of a small concentration of $\mathrm{H}_{2}^{\neq}$in a predominantly $\mathrm{H}^{+}$ plasma. 
In the following sections two different consequences of the hybrid resonance are discussed: enhancement of the second harmonic cyclotron damping and linear mode conversion.

Enhanced Cyclotron Harmonic Damping

The two-ion hybrid resonance layer lies between the fundamental cyclotron resonance layers of the two ion species. If one species is a minority, the hybrid layer lies close to the cyclotron layer of that species. In such a case the strength of the hybrid resonance is modified by the fundamental cyclotron damping due to the minority species. The case of a deuterium plasma with a small concentration of protons represents a more complicated situation, because of the degeneracy of the fundamental proton and second harmonic deuteron cyclotron resonances. Here, the second harmonic damping may be profoundly influenced by the proximity of the hybrid resonance. This can be seen qualitatively by examining the polarization of the wave E-field near the hybrid resonance in the cold plasma approximation. From Eq. (12) the left- and right-handed components of the electric field normalized by $i E_{y}$ is given by,

$$
\frac{E_{ \pm}}{i E_{Y}}=I \pm \rho
$$

At a two-ion hybrid resonance $(\mathrm{H}=0) \mathrm{E}_{\mathrm{x}} \rightarrow \infty$ and the field is linearly polarized in the $x$-direction (i.e., the waves are electrostatic). Both $E_{+}$and $E_{-}$are infinite. At a hybrid cut-off $(G=0) \rho=+1$ and the field is purely left-hand polarized. The presence of the hybrid resonance produces another special pisint at which the polarization 
takes on a particular value: At a point where $D=0, \rho=0$ and the field is linearly polarized in $y$-direction. Finally, at a cyclotron resonance $(L=\infty), \rho=-I$ and the polarization is purely right-handed. These variations of polarization are shown in Fig. 7 along with the direction of the E-field vector. Over a small distance from the hybrid to the cyclotron resonance, therefore, $E_{+}$component varies from infinity to zero. This is in contrast to the situation without any resonant species in which $\mathrm{E}_{+}$ component is but a small fraction of the total field and varies only slowly over the region of the fast wave propagation. We now recall that the strength of the second harmonic heating is proportional to $\mid$ grad $\left.E_{+}\right|^{2}$ and that the cyclotron damping takes place in the cyclotron layer of small but finite thickness due to thermal effects. We are then led to consider the possibility that the second harmonic damping strength may be considerably greater in two-ion plasmas than in one-ion plasmas which have no hybrid resonance. Of course, these same thermal effects reduce the strength of the hybrid resonance and $E_{+}$there is finite. The question is how strong is the influence of cyclotron damping in reducing the strength of hybrid resonance and how strong is the influence of the hybrid resonance in modifying the field structure and hence the strength of the cyclotron damping. None of the theoretical works mentioned in the previous section obtained explicit solutions to a boundary value problem to examine the field structure inside the plasma in the presence of the hybrid resonance. This section presents some preliminary results from an analysis in a simple slab geometry. The geometry 
and the coordinate system are shown in Fig. 8. A Tokamak-like field variation $\left(B_{0}=B_{0} R_{0} / R\right)$ and parabolic electron density and ion temperature profiles are assumed. Numerical examples given below are for $\mathrm{R}_{0}=0.9 \mathrm{~m}$, the slab half-width, $\mathrm{a}=0.17 \mathrm{~m}$ and the central electron density, $n_{e o}=2.5 \times 10^{19} / \mathrm{m}^{3}$. The origin of the $(x, y, z)$ coordinate system is located at the center of the slab.with $x$-axis in the direction of inhomogeneity pointing toward the high field side. B-field is in the positive $z$-direction and all variations in $y$-direction are ignored. The quantity $\xi=k_{0} x=(\omega / c) \times$ is a normalized coordinate and its origin may be left arbitrary at the moment.

In this analysis thermal effects are treated in lowest order. All non-resonant species (e.g., deuterons, electrons, etc.) are treated in cold plasma approximations and the resonant species (e.g., protons) include thermal effects only in motion along the B-field. The cold electron approximation may be justified for small present-day Tokamaks, but may he poer for larger and huller plasmas. Inclusion of the electron thermal effects would further reduce the strength of the hybrid resonance through electron transit time and Landau damping. Finite Larmor radius effects are ignored. Hence the results include fundamental cyclotron damping due to resonint species but not the harmonic damping due to nonresonant species. The latt.er will bo octimated ili lle same spirit as STIX's analysis (18) from the calculated field structure. The resonant species' contributions to the dielectric tensor are twofold: their real part is responsible for the hybrid resonance and the imaginary part for the cyclotron damping. The dielectric 
tensor under these conditions (see, e.g., STIX(24), p 192) can be shown to have the same form as that of a cold plasma in that xz; $y z, z x$ and $z y$ components are zero. The remaining terms have both real and imaginary corrections. The differential equation for wave propagation then reduces to the following form.

$$
\begin{aligned}
& \frac{d^{2} E_{y}}{d \xi^{2}}+n_{\xi}^{2} E_{y}=0 \\
& n_{\xi}^{2}=\frac{) k_{R}-n_{\|^{2}}\right)\left(k_{L}-n^{2} \|^{2}\right.}{\left(k_{\perp}-\dot{n}^{2}\right)} \\
& \rho_{\xi} \equiv \frac{E_{x}}{i E_{y}}=\frac{k_{x}}{k_{\perp}}
\end{aligned}
$$

where $\mathrm{n}_{\xi}$ and $\rho_{\xi}$ are complex perpendicular refractive index and polarization. These latter two quantities reduce respectively to Eqs. (11 and 12) in the low temperature limit. $K_{R^{\prime}} K_{L^{\prime}}, K_{\perp}$ and $\mathrm{K}_{\mathrm{X}}$ are warm plasma dielectric tensor elements and are given by,

$$
\begin{aligned}
& \mathrm{K}_{\mathrm{R}}=\mathrm{R} \\
& \mathrm{K}_{L}=1-\sum_{\sigma} \frac{\Omega^{2} \mathrm{p} \sigma}{\left(1-\varepsilon_{\sigma} \Omega_{\sigma}\right)}+\sum_{\gamma}{ }^{2} \Omega^{2} \mathrm{p}_{\gamma}\left(\frac{\mathrm{v}}{\mathrm{v}_{\gamma}}\right) \mathrm{z}\left(\alpha_{-1}^{\gamma}\right) \\
& \mathrm{K}_{\perp}=\frac{1}{2}\left(\mathrm{~K}_{\mathrm{R}}+\mathrm{K}_{\mathrm{L}}\right) \\
& \mathrm{K}_{\mathrm{X}}=\frac{1}{2}\left(\mathrm{~K}_{\mathrm{R}}-\mathrm{K}_{\mathrm{L}}\right) \\
& \alpha_{ \pm 1}^{\gamma}=\left(\frac{v}{v_{\gamma}}\right)\left(1 \pm \Omega_{\gamma}\right)
\end{aligned}
$$


where summations $\sum_{\sigma}^{\prime}$ and $\sum^{\prime \prime}$ are over all non-resonant and all resonant species, respectively, and $\mathrm{Z}$ is the plasma dispersion function. $K_{R}$ has no significant resonant species contributions and is identical to its cold plasma counterpart (Eq. 16).

Since $\mathrm{K}_{\perp}$ is now complex, the denominator of the right hand side of Eq. (24) does not necessarily vanish. We define existence of strong hybrid resonances by vanishing of the real part of the denominator,

$$
R_{e}\left(k_{\perp}-n_{\|}^{2}\right)=0
$$

The maximum value of the real part of the dispersion function, $z\left(\alpha_{-1}^{\gamma}\right)$, is 1,082 (see, e.g., STIX (24), Fig. 8-1). The condition can then be cast into the following form:

$$
\Omega_{\mathrm{p} \gamma}^{2} \geq \frac{2}{1.082}\left(\frac{\mathrm{v}_{\mathrm{\gamma}}}{\mathrm{v}_{\|}}\right)\left\{\sum_{\sigma}^{\text {ion }} \frac{\Omega^{2} \mathrm{p} \delta}{\left(1-\Omega_{\mathrm{p}}^{2}\right)}\right\}+\mathrm{R}_{\mathrm{e}}\left(\mathrm{n}_{\|}^{2}\right)
$$

where summation $\sum_{\sigma}^{\text {ion }}$ is now over non-resonant ions only. For a $\mathrm{D}-\mathrm{H}$ plasma the condition in terms of proton concentration (with respect to electron density) is given by,

$$
C_{H} \equiv \frac{n_{H}}{n_{e}} \geq \frac{2}{1.082} C_{D}\left(n_{||} \frac{v_{H}}{C}\right)\left\{\frac{1}{1-\Omega_{D}^{2}}+\frac{n_{D}^{2}}{\Omega_{p D}^{2}} \mid\right.
$$

From this equation we see that for any given value of $\mathrm{C}_{\mathrm{H}}$ the strong resonance disappears, if: (1) $\mathrm{n}_{\mid}$becomes large, (2) density becomes small or (3) temperature becomes high. The first case was also noted by JACQUINOT, SCHARER and MCVEY (32). For $\mathrm{T}_{i}=200 \mathrm{eV}$, 
$\mathrm{n}_{\mathrm{e}}=2.5 \times 10^{19} / \mathrm{m}^{3}$ and $\mathrm{n}_{\|}=20$, Eq. (33) gives $\mathrm{n}_{\mathrm{H}} / \mathrm{n}_{\mathrm{e}}>5 \%$. We are primarily interested in cases in which $C_{H}$ is comparable to or smaller than the limiting value given by this equation. The resonance is therefore weak and the perpendicular wavelength remains of the same order as the parallel one. This provides justification for neglecting $x z, y z, z x$ and $z y$ elements of the warm plasma dielectric tensor.

Vanishing of the complex electric field, $E_{y}$, at conducting boundaries provides boundary conditions for Eq. (23) . Complex eigenvalues for $\mathrm{n}_{\|}$are to be found. For each value of $\mathrm{n}_{\|}$the field structure can then be calculated. The eigenvalue search and field calculations were done numerically and some examples. for conditions that may approximate high density ATC experiments are shown in Fig. (9). The top figures are variations of $\mathrm{E}_{\mathrm{x}}$ across the slab for three different values of magnetic field: one for which the proton cyclotron resonance is located at the center and two others for which the resonance is displaced by $6 \mathrm{~cm}$ on either side of the center. The lower figures are variations of the left-hand polarized E-field for the corresponding three cases. Locations of the ion cyclotron (ICR) and two-ion hybrid (HBR) resonances are indicated in these figures. (The latter for these weak resonances is defined as the location of the minima of the real part of $k_{\perp}-n_{\|}^{2}$.) Effects of weak hybrid resonances appear as small bumps on $E_{x}$-curves $\left(E_{y}\right.$ is little affected). The effects are stronger on the low field side of the axis, evidently as a result of the fact that the field and density gradients are in the same direction there. However, influence of the hybrid resonance on the $E_{+}$-field is significant. Compare $C_{H}=0$ and 38 cases shown in the 
lower central figure. This is a consequence of the fact that the field polarization is strongly altered by the hybrid resonance. Particularly noteworthy here is the appearance of a very steep gradient of the $\mathrm{E}_{+}$-field over a narrow region between the cyclotron and hybrid resonances. In the case shown in the lower left figure the gradient in this region is enhanced by one order of magnitude over the value obtained without hybrid resonance. Then, in the spirit of STIX's analysis(18) discussed earlier, the deuteron second harmonic damping could be enhanced by two orders of magnitude. (For that matter, any species whose cyclotron harmonic resonance falls in this region would have greatly enhanced effects on wave damping.) With such great enhancement this mechanism could possibly account for the observed strong damping of the fast wave. We also note that the region of cyclotron damping must extend on the high field side considerably further than indicated in Fig. $2(a)$. In order to obtain the precise enhancement factor, the damping strength must be integrated over the width of the resonant region, but this has not yet been done here. Also omitted from consideration is a possibility that this strong harmonic damping might reduce the strength of the hybrid resonance further which in turn would moderate the harmonic damping strength. The same can be said of the electron transit time and Landau damping. Nonetheless, it is clear that the cyclotron harmonic damping strength in a two-ion plasma cannot be evaluated properly without giving considerations to the effects of the hybrid resonance.

The normalized damping length computed from the imaginary part of the eigenvalue is plotted in Fig. 10 as a function of the 
major radius location of the proton cyclotron resonance $\left(\mathrm{C}_{\mathrm{H}}=3 \%\right)$. It must be noted that this represents only the minority heating (proton fundanental cyclotron damping). The curve again exhibits an asymmetry with respect to the plasma center. As a comparison a few cases without the hybrid resonance (the real part of the resonant species contribution to $K_{\perp}$ in $\mathrm{Eq} \cdot(27)$ is set equal to 0 ) are also shown in the same figure (labeled as w/O HBR). Beyond enhancing the minority cyclotron damping by a factor up to two or so, the effect of the hybrid resonance is to accentuate the asymmetry. In Fig. (1i) the damping length is plotted as a function of the proton concentration. The damping length is well over unity for the range of proton concentration presented in the figure. Thus even with the enhancement by the hybrid resonance the minority cyclotron damping alone does not seem to be able to account for the observed strong damping and disappearance of toroidal eigenmodes.

Damping Due to Mode Conversion

In the opposite limit of high resonant species concentrations the hybrid and cyclotron resonance layers are well removed from each other and the cyclotron damping in the vicinity of the hybrid resonance layer may be ignored. Then the governing wave equation is again Eq. (23), but with $\mathrm{n}_{\xi}$ replaced by its real counterpart, $\mathrm{n}_{\perp}$ (Eq. (11) . The resulting physical model is identical to the one employed by JACQUINOT, MCVEY and SCHARER (32) and is considerably simpler than the model used by PERKINS (33) who treated a more general case with $k_{y} \neq 0$. Here, we derive some analytical expressions 
for BUDDEN's parameters and wave damping strength. For this purpose it is now convenient to fix the origin of the $\xi$-coordinate at the hybrid resonant layer. The equation then takes the form,

$$
\frac{a^{2} E_{y}}{d \xi^{2}}+\left(\frac{\beta^{2}}{\eta^{2}}+\frac{\beta}{\xi}\right) E_{y}=0
$$

Budden's parameters, $\beta$ and $n$, can be obtained by expanding the hybrid resonant function, $\mathrm{H}$ (Eq. 15), into a Taylor series and retaining only the first term:

$$
\begin{aligned}
& B=\frac{\left(\mathrm{R}-\mathrm{n}^{2}\right)^{2}}{-\left.\frac{\mathrm{dH}}{\mathrm{d} \xi}\right|_{\xi=0}}=\frac{\left(\mathrm{k}_{\mathrm{o}} \mathrm{R}_{\mathrm{HB}}\right)\left(\mathrm{R}-\mathrm{n}_{U^{2}}\right)^{2}}{2\left\{\left(\mathrm{n}^{2}-1\right)+\sum_{i} \frac{\Omega^{2} \mathrm{pi}}{\left(1-\Omega_{i}^{2}\right)^{2}}\right\}} \\
& \eta^{2}=\frac{B^{2}}{2\left(R-n_{\|^{2}}\right)} .
\end{aligned}
$$

The summatinn in Eq. (J5) is over all inns. R $\mathrm{R}_{\mathrm{HB}}$ is Lle major radius location of the hybrid layer and $R, \Omega_{\mathrm{pi}}$ and $\Omega_{i}$ are to be evaluated there. The quantity $B_{0}^{2} / n^{2}$ represents the asymptotic value of $n_{\perp} 2$ and $-n^{2} / \beta$ the thickness of the evanescent layer between the hybrid resonance and cut-off (Fig. (12)). As shown by BUDDEN (29) the waves incident from the high field side are partially transmitted and partially absorbed. The transmission coefficient and the fraction of energy absorbed are given respectively by, 


$$
\begin{gathered}
\left|\mathrm{T}_{\mathrm{H}}\right|=\mathrm{e}^{-\frac{1}{2} \pi n} \\
A_{\mathrm{H}} \equiv 1-\left|\mathrm{T}_{\mathrm{H}}\right|^{2}=1 \mathrm{e}^{-\pi n} .
\end{gathered}
$$

The waves incident from the low field side are partially transmitted, reflected and absorbed with transmission and reflection coefficients and the fraction of energy absorbed given respectively by,

$$
\begin{gathered}
\left|T_{L}\right|=e^{-\frac{1}{2} \pi n} \\
\left|R_{L}\right|=1-e^{-\pi n} \\
A_{L} \equiv 1-\left|T_{L}\right|^{2}-\left|R_{L}\right|^{2}=e^{-\pi n}\left(1-e^{-\pi n}\right)
\end{gathered}
$$

It was pointed out by STIX (35) that the important quantity, $n$, is the thickmess of the evanescent zone measured in the units of the asymptotic wave number. In Fig. $13(a)$ the Budden's parameters are plotted as a function of proton concentration. Note that $n$ and hence the absorption is small unless, the proton concentration exceeds 10\%. (Here, the proton cyclotron layer is fixed at the plasma center and therefore the hybrid layer moves toward the low density plasma edge as $C_{H}$ increases. Fall-off of $\beta$ and $\eta$ at high concentrations is due to lower densities at the hybrid layer. The latter is nearly $10 \mathrm{~cm}$ away from the cyclotron layer at $\mathrm{C}_{\mathrm{H}}=20 \%$.) 
Variation of the parameters as the cyclotron layer is moved across the plasma region is shown in Fig. $13(\mathrm{~b})$ for $\mathrm{C}_{\mathrm{H}}=3 \%$. The asymetry characteristic of the hybrid resonance can again be seen.

We may regard the boundary value problem treated in the previous section as damping of a plane wave bounding back and forth between two retlecting boundaries while also progressing in z-direction (Fig. 14). Starting, e.g., from the high field side boundary, the energy remaining in the waves after one complete transit (back and forth crossing the resonant zone twice) is given by $\left[1-\left(1-A_{H}\right) A_{L}\right]$. If the wave amplitude e-folds after $m$ such transit, then $m$ is given by,

$$
m=\frac{-2}{\ln \left\{1-\left(1-A_{H}\right) A_{L}\right\}}
$$

For each transit the waves travel in parallel direction a distance $\mathrm{d}=4 \mathrm{an}_{\|} / \overline{\mathrm{n}}_{\perp}$ where $\overline{\mathrm{n}}_{\perp}$ is to be averaged over the slab with $2 \mathrm{a}$. The normalized damping length can then be calculated crudely (In this analysis the fate of the wave reflected at the hybrid layer is ignored.) from,

$$
\frac{L_{D}}{L}=\frac{m d}{L}=\frac{2}{\pi} \quad\left(\frac{a}{\bar{R}_{0}}\right)\left(\frac{n_{\Perp}}{\bar{n}_{\perp}}\right) m
$$


Using $\exp (-\pi n)=0.6$ from Fig. $13(\mathrm{~b})$ and $\bar{n}_{\perp} / n_{\|}=3 / 2(*)$ we obtain $\mathrm{L}_{\mathrm{D}} / \mathrm{L}=2$ which is close to the value for minority heating obtained at the end of the previous section. Thus in order to explain experimental observations $\left(L_{D} / L<1\right)$ in terms of mode conversion damping we must assume a proton concentration substantially greater than $3 \%$ used here.

Resonant Layers in Tokamaks

Tokamak plasmas contain in general a variety of impurity ions in different states of ionization. Therefore, for any set of operating conditions there may be many impurity species with their fundamental cyclotron resonance layer within the plasma region. The result would be the existence of a multitude of hybrid resonances between these ions and the majority ion species. It must be remembered in this context that the minority species concentration enters into equations as ion plasma frequency. A small concentration of multiply charged impurities can therefore have important effects on wave damping and heating. For convenience in identifying these ions for any given operating ranges, a chart in Fig. 15 is prepared. Several plasma ion species and commonly found light impurities are indicated along the $y$-axis, which is the dimensionless mass-to-charge ratio. The $x$-axis is

(*)For this small concentration $\mathrm{n}_{\xi}^{2}$ is strongly complex near the hybrid layer. The maximum value $\xi_{f}$ its real part is of the order of 600, instead of the asymptotic value 2200 calculated from BUDDEN's parameters. If we use an average value of $\overline{\mathrm{n}}_{\perp}^{2}=450$, then we obtain $L_{D} / L=4.5$. 
either frequency, magnetic field or major radius location all normalized by their respective values for the proton cyclotron resonance. The $n$-th harmonic resonant condition is satisfied for $h=n / x$. The location or the range for the resonance in terms of frequency, field or radius can be conveniently found from this chart. For example, for TFTR $\left(R_{0}=2.5 \mathrm{~m}, a=0.85 \mathrm{~m}\right)$ operating at $B=4.5 \mathrm{~T}$ with rf frequency of $\omega=2 \pi \times 25 \mathrm{MHz}$, what kind of resonances can be found in the plasma? We first calculate the normalizing factor. At $25 \mathrm{MHz}$ a proton is resonant at 1.64T. Therefore, $\mathrm{R}\left(\Omega_{\mathrm{H}}=1\right)=2.5 \times 4.5 / 1.64=6.86 \mathrm{~m}$. The inner and outer edges of the plasma are at $1.65 \mathrm{~m}$ and $3.35 \mathrm{~m}$. Then, $R / R\left(\Omega_{H}=1\right)=0.241-0.488$. This range is indicated in the figure by a circle labeled TFTR. If the device is run with a D-T plasma, we see that between the triton and deuteron resonances there are many light impurity ions resonant at their fundamental frequencies all with associated two-ion hybrid resonances with deuterons. Thus a study of physics of hoating uiler such conditinns would be exlremely complicated. In this regard use of a hydrogen and ${ }^{3} \mathrm{H}_{\mathrm{e}}$ mixture suggested by PERKINS(33) is interesting: The second harmonic of ${ }^{3} \mathrm{H}_{\mathrm{e}}^{++}$cyclotron frequency is not degenerate with the fundamental proton cyclotron frequency. Furthermore, there are no other elements between the fundamental cyclotron frequencies of the two elements to complicate the picture (Fig. 15). Wavo damping experlments with this mixture should help resolve many of the questions that arose from $\mathrm{D}-\mathrm{H}$ experiments. Another example shown in the figure is for PLT $(R=1.3 \mathrm{~m}, \mathrm{a}=.4 \mathrm{~m})$ operating at $3.5 \mathrm{~T}$. What are the resonances that come into the plasma, if rf frequency is varied from 40 to 
$60 \mathrm{MHz}$ ? The plasma edges are at 0.9 and $1.7 \mathrm{~m}$ and the fields there are 5.06 and 2.68T, respectively. The normalization factor $\omega_{H}$ at these values of $\mathrm{B}$ are $2 \pi \times(77.1$ and $40.8 \mathrm{MHz})$, respectively. We therefore find the range to be $\omega / \omega_{\mathrm{H}}=0.99-1.47$, which is shown in the figure by a range bar labeled PLT. Similarly, the range of resonant species that crosses the inner edge of ATC plasma, if. the B-field is varied by $0.4 \mathrm{~T}$ around the central value of $1.5 \mathrm{~T}$ is indicated in the figure. 
PART II - ION HEATING EXPERIMENTS IN ATC

Ion heating experiments at high rf power levels have been conducted earlier in several devices $(7,11,12,13,15)$. Although encouraging results were obtained in that the ion energy distribution could be significantly affected by the ICRF heating, ion temperature diagnostics in these experiments were limited and the nature of the observed heating was not thoroughly studied. Before a conclusion is drawn on the ability of ICRF in heating Tokamak plasmas, it must be decided whether rf energy is deposited in: (1) the core or edge of the plasma, (2) the bulk or tail of the ion distribution, (3) perpendlcular or parallel degree of freedom and (4) the majority or minority ion species. In the latest experiments in ATC (17). the nature of the observed heating was investigated in detail using several diagnostic techniques. Another important difference in the ATC experiments is that the rf heating prise was applied over a period considerahty longer than various ion relaxation times (equipartition, deflection times, etc.) and the: heating at the pulse end could be more reliably assessed.

Details of the ITC device can be found elsewhere (BOL, et al. (36)) The ex:periments are conducted in deuterium plasmas at central. electron densities of $0.5-3.5$ (typic.ally 2.5 ) $\times 10^{19} / \mathrm{m}^{3}$ and toroidal magnetic induction typically of 1.7 'resla. The antenna is made of 135-degree arc-shaped copper strip, $L^{\prime} \times 1 / 4 "$ in cross-section, encased in a ceramic sheath (MACOR machinable glass ceramic) and is protected from direct contact with the plasma by a pair of stainless-steel bumper limiters (Fig. 1). The limiters are 
separated by a distance of $20 \mathrm{~cm}$ in the meridian plane (The indentations in the limiters are for the purpose of accommodating other experiments). The terminating capacitor is tuned to bring the virtual ground to the antenna mid-point. The impedance matching is accomplished through the tuning capacitor and two stub. tuners (not shown in the figure). The rf power level (maximum peak power approximately $200 \mathrm{~kW}$ ) droops considerably during the pulse and all values quoted below refer to the time average. The maximum rf energy input into the plasma is limited by occurence of magnetohydrodynamic (MHD) oscillations that often lead to plasma disruption. At high rf power levels titanium gettered surfaces (STOTT, DAUGHNEY and ELLIS (37)) are used to avoid the disruption. When $\mathrm{m}=2$ MHD oscillations are excited, various indications of deterioration of ion energy confinement are observed (21). In all of the heating experiments reported below the rf power and plasma parameters are adjusted to avoid m=2 MHD oscillations. However, the rf generator frequency (fixed at $25 \mathrm{MHZ}$ corresponding to the seconci harmonic deuteron cyclotron frequency at $1.64 \mathrm{~T}$ ) is matched well to the ATC device and tire experiments can be conducted under favorable discharge conilitions. Principally for this reason, the maximum $r f$ energy input is one order of magnitude greater than for thc ST experiments (7).

Ion Temperature Diagnostics

The principal ion temperature diagnostic is a three-channel charge exchange (CX) neutral particle analyzer aimed perpendicularly ("perpendicular temperature") at the plasma. There is also a 
one-channel cx analyzer viewing the plasma in the direction tangent to the torus ("parallel temperature"). These analyzers are not capable of discriminating mass differences and hence their signals contain contributions not only from deuterons but also from minority protons. An oscillograph of the perpendicular analyzer output and an example of energy spectrum are shown in Fig. 16. At high energies the spectrum is distorted by the presence of a "tail" created by the rf fields. Interpretation of the cX spectra is complicated, because the high energy part of the spectra that would ordinarily be used to determine the ion temperature in the plasma interior (PARSONS and MEDLEY :38) would now give temperatures too high as a measure of the thermal content of the ion species. The CX spectrum for the rf-on case in Fig. 16 is fitted with two straight lines by the least-squares method. The fit in the low energy part (900-1950 ev) is used to determine "body temperature" and the one in the high energy part (3000-4500 ev) "tail temperature". The body temperatures are used to evaluate the amount of heating.

Impurityr ions of given states of ionization are concentrated within narrow annular regions in Tokamak discharges determined primarily by the electron temperature: Moreover, these ions are strongly coupled thermally to the majority deuterons throughout frequent colisions. Thus a radial lon temperature profile can be determined by measurement of impurity ion temperatures. The latter may be obtained through spectroscopic Doppler width measurements of line emissions from impurity ions. It should be noted that the Doppler profile is determined by ions in the thermal 
(not the tail) part of the distribution. Temperatures of OVII, $\mathrm{CV}$ and CIV (see Fig. 17(a)), near the end of the rf heating pulse $\left(\mathrm{P}_{\mathrm{rf}} \simeq 72 \mathrm{~kW}\right)$ are measured by a monochrometer equipped with a vibrating LiF plate, which rapidly scans the Doppler profile (SUCKEWER and HINNOV (39)). The radial locations of the annuli determined from the diffusion velocities and ionization times of these impurities (39) are indicated by the horizontal bars and the extent of shot-to-shot variation by the vertical bars. A factor of two uncertainty in the values of the rate constant and diffusion velocity would affect the radial positions by 1 ess than $1 \mathrm{~cm}$. These impurity temperatures are compared with the cx body temperature. Detailed studies of the Cx signal source function, using the neutral density profile calculated by STOTT (40), indicate that the measured $\mathrm{Cx}$ temperature represents an average over the inner half of the plasma. A probable profile suggested by these measurements is shown in Fig. $17(\mathrm{a})$ by a solid curve and a profile similarly determined for a discharge without heating by broken line. All temperatures in the figure, except that of CIV, increase in response to application of the hecting pulse.

The narrower profile with rf (Fig. $17(\mathrm{a})$ ) is believed to be a result of peripheral cooling due to additional influx of impurities caused by the heating pulse. The sane influx is thought to be responsible for the observed electron density increase which is typically about $30 \%$ but which may vary greatly depending upon plasma conditions. (The fractional increase is smaller at higher 
densities.) Intensities of carbon impurity lines increase substantially during the heating pulse. However, it is not clearly understood at present whether this influx alone is responsible for the observed density increase.

The third method used to determine ion temperature makes use of neutron counting ("neutron temperature"). Upon termination of the rf pulse, the plasma is compressed to higher temperatures and densities. The neutron temperatures are determined assuming a dewteron defficiency of 0.5 and are compared with the CX body temperatures in Fig. $17(\mathrm{~b})$. Because of high densities $\left(>10^{20} / \mathrm{m}^{3}\right)$ and consequent short relaxation times $(<1 \mathrm{~ms})$, the ion distribution should become nearly Maxwellian within a few milli'seconds after compression. Thereafter, neutron production by high energy tail ions should be negligible. Moreover, the cx ion temperature in the plasma interior can be determined more reliably using higher energy part of the spectrum. Reasonable agreement between the neutron and $\mathrm{CX}$ temperatures is demonstrated in the figure. The temperatures reached after compression are also in agreement with predictions of the adiabatic toroidal compression theory (36) indicated in the figure by the lines marked A.T.C.

The CX diagnostics, which can be used in both gettered and ungettered discharges with or without compression, are the most convenient technique and are used to determine ion heating under various conditions. A typical example of the temporal variation of the perpendicular body temperature is shown in Fig. 18(a) for discharges with and without an rf pulse $\left(P_{r f} \simeq 72 \mathrm{~kW}\right)$. After 
termination of the heating pulse the temperature relaxes to the value without rf in a time consistent with the calculated ionenergy replacement time. Thus, the rf heating causes no significant change in the ion-energy confinement, and the heating is attributable to the rf power input and not to a change in ohmic heating caused by impurity influx (7). The parallel body temperature exhibits a somewhat higher value, but its temporal evolution is essentially the same.

Fig. $18(\mathrm{~b})$ shows the temperature increase attributable to a 10-ms rf heating pulse. The rf power plotted along the abscissa is normalized by the total number of ions in the device (calculated on the basis of a parabolic ion density distribution). A broken line is drawn through the experimental points by the least-squares method to indicate an upward trend of the temperature rise with increasing rf power per ion. The maximum heating results in a $200-\mathrm{eV}$ increase in the ion temperature at an average rf power of $145 \mathrm{~kW}$. An ion heating efficiency may be defined as the ratio of the measured to theoretical ion energy increase at the end of the heating pulse. The theoretical value taking account of the finite ion-energy confinement time, $\tau$, is given by $\tau P(1-\exp -t / \tau)$. Here $\mathrm{P}$ and $t$ are the rf power and pulse length, respectively. In calculating the ion energy increase radial profiles of the ion density and temperature are assumed to be parabolic. The cx body temperature is taken to be the peak temperature (This may be an underestimate -see Fig. $17(\mathrm{a}))$. The efficiency ranged from 10 to $40 \%$. 
Fast Ion Slowing-Down Process

Additional information on the location of the observed heating can be obtained from detailed examinations of the CX signals. If the heating were confined within the plasma periphery, the dominant ion loss would be through uncontained drift orbits with a time constant comparable to the bounce time $(\leqslant 0.1 \mathrm{~ms})$ around the banana orbits. The CX signals; especially c.t high energies (say, 3 $4.5 \mathrm{keV}$ ), would then decay rapidly after termination of the heating pulse. On the other hand, if the observed signals originate from the central core (say, r/a $<0.3$ ) the governing fast-ion depopulating process is the slowing-down due to the ion and electron drag. Thus comparison of the observed signal decay time with theoretical predictions provides a clue to the origin of the heated ions that undergo charge exchange and are intercepted by the analyzer.

We consider relaxation of a smal.I number of fast ions slowing down on a steady background plasma. The fast ion distribution, f, is assumed to remain isotropic throughout the process. This assumption is consistent with experimental observations: The tail part of the ion distribution tends to be more or less isotropic (especially at high densities), presumably due to fast deflecting collisions with impurity ions. A continuity equation in the velocity space is then,

$$
\frac{\partial f}{\partial t}+\nabla_{v} \quad f \frac{\partial \vec{v}}{\partial t}=0
$$

Writing out the operator in the spherical coordinates and applying the isotropy assumption leads to, 


$$
\frac{\partial f}{\partial t}+\bar{\varepsilon}^{1 / 2} \frac{\partial}{\partial \varepsilon} \cdot\left(f \varepsilon^{1 / 2} \frac{d \varepsilon}{d t}\right)=0
$$

where $\varepsilon=1 / 2 m_{i} v_{i}^{2}$ is the kinetic energy of an ion. The rate of energy loss due to the electron and ion drag is given by STIX(41),

$$
\frac{d \varepsilon}{d t}=-\frac{2 \varepsilon}{t_{s}}\left[1+\left(\frac{\varepsilon_{c}}{\varepsilon}\right)^{3 / 2}\right]
$$

where $\varepsilon_{C}$ and $t_{s}$ are respectively the critical energy and slowingdown time on electrons. Note that the critical energy is of the order of $5 \mathrm{keV}$ and hence both the electron and ion drag terms are. important in the present situation. Upon substitutions, $\tau=3 t / t_{S}$ and $W=\left(\varepsilon / \varepsilon_{C}\right)^{3 / 2}$, the equation takes the form,

$$
\frac{\partial f}{\partial \tau}-\frac{\partial}{\partial w}[(I+w) f]=0
$$

The initial condition is,

$$
f(0, w)=\phi(w)
$$

where $\phi(W)$. is an arbitrary function of W.:Eq. (46) can be integrated by an elementary method (see, e.g., HILDEBRAND(42)) and the solution is given by,

$$
E(\tau, w)=e^{\tau} \phi\left[(1+w) e^{\tau}-1\right]
$$


The tail part of an ion distribution measured at the end of an re: pulse is represented by a polynomial. It is:used as an initial distribution: (Eq. 48) and the fast ion relaxation is calculated from Eq.. (49). In Fig. 19 (a) the CX signal decay process inferred: from the calculated fast ion relaxation is compared: to the experimental observation at one value of energy. In: Fig\%. I9(b); theoretical and experimental half-widths (CX signal decay time to half-height) are shown for a range of energies. Good agreement shown: in these figures is a further indication that the ion heating occurs in. the central core of the plasma.

Influence of Proton Impurity

Examination of the time. evolution of the CX signals and temperature during the early phase of the heating pulse (see Figs. I6 ard $18(a))$ reveals some anomalies: Initially they rise rapldIy; sonetimes exhibiting a small overshoot, and come to saturation well before the time expected from the ion energy replacement time. These anomalies can be best explained in terms of spurlous effects of the impurity protons whlch cannot be discriminated by the $C X$ analyzers uged in the present experiments. The impurity protons have equipartition times (SPITzER(43)) short (0.1) ms at body temperatures and $2 \mathrm{~ms}$ at tall temperatures) compared to the Ion energy confinement time (5-11 ms) and their main energy 1008 18 rapld power transfer to the deuterons. If the protons aboorb rf power alrectily, then they may be heated, due to the1r small thermal Inertia, to temperatures much higher than deuterons and within a few eqilpartition times quickly come 
to a saturation temperature determined by the instantaneous power level. During this early phase the CX signals contain disproportionately large contributions from the protons despite their small concentration and the temperature determined from these signals is an overestimate of the true thermal energy content of the ion species. The situation changes, however, as the deuterons heat up and the proton temperature falls due to the drooping rf power, and at the end of the pulse the proton contribution becomes negligibly small. The fact that the cx body temperature decays with a time scale consistent with the ion energy confinement time rather than the much'shorter equipartition time is evidence that the non-thermal protons have negligible influence in the $\mathrm{CX}$ body temperature determination at the end of the heating pulse. It should also be noted that, because the heating pul.se is much longer ( $10 \mathrm{~m}: \mathrm{s}$ ) than the equipartition time, the protons would give up to the deuterons most of the energy they received from the $\mathrm{rf}$ fields. In order to test the validity of this hypothesis a model for the equipartition process is constructed based upon equations similar to those given by SPITZER (44) but including effects of the finite energy replacement time and the rf power input. Numerical integration of these non-linear equations indicates that the anomalous features mentioned earlier can be explained by this model.

From measurements described in the preceding sections it appears reasonable to conclude that the observed heating represents increase in thermal content of the majority deuterons. However, an important question still remains unanswered in a satisfactory 
manner: Do the deuterons themselves absorb power from the rf wave field or are they heated indirectly through energy exchange with protons? In experiments in the T-4 device $(12,13)$, in which a mass-discriminating $\mathrm{CX}$ analyzer was used, heating of protons by the wave field was clearly observed, while deuteron cx spectra were affected only through peripheral heating by the near field of the antenna. In these experiments, however substantial rf power could be coupled into the plasma only during occurence of toroidal eigenmode resonances (SOKOLOV (45)). The duration of each resonance (a few hundred microseconds) was much shorter than either the deuteron-on-proton equipartition time or the deuteron heating time. While these experiments clearly establish direct power absorption by the protons, no definite conclusion can be drawn on the behavior of the deuterons. Further experiments at sustained high pwer level using mass-discriminating analyzers would help clarify the details of the heating process.

\section{CONCLUDING REMARKS}

There are a number of experimental indications which support the view that the two-ion hybrid resonance plays an important role in ICRF heating of a deuterium plasma. They are: (1) damping strength in deuterium plasmas at the second harmonic deuteron cyclotron frequency, which is much stronger than expected, (2) damping strength in hydrogen plasmas at the second harmonic proton cyclotron frequency, which is weak and roughly in line with expectations, (3) dependence of damping on amount of hydrogen gas injected, (4) asymmetry of damping curve, (5) asymmetry of charge exchange loss. 
From theory we find: (6) Inclusion of two-ion hybrid resonance makes various damping mechanisms including proton cyclotron, deuteron second harmonic and mode conversion damping all asymmetric with respect to the plasma center. (7) Proton cyclotron damping appears insufficient to explain the observed damping strength. (8) Mode conversion damping appears to be sufficiently strong to explain experimental results, provided that proton concentration is high. (9) Enhanced second harmonic damping due to proximity of two-ion hybrid resonance may be strong enough to account for the experiments at modest values of proton concentration.

From these observations it appears reasonable. to conclude that the two-ion hybrid resonance has important influence on ICRF heating of a deuterium plasma. However, details of the mechanism with which the resonance affects the wave damping seem not yet clearly established. As a consequence an important. question whether deuterons absorb energy directly from the rf wave field or indirectly through protons is not yet adequately answered. An explanation based upon a mode conversion at the hybrid resonance appears to be promising. However, as indicated by the preliminary analysis in this paper, there may be another mechanism (enhanced second harmonic damping) also caused by the hybrid resonance that is compatible with experimental observations. The mode conversion is expected to be strong at high resonant species concentration ( $>10 \%$ ), while the enhanced cyclotron damping is strong at more modest values of the concentration (< 5\%). The latter is peculiar only to deuterium plasmas (with proton impurity) under the second harmonic heating conditions. 
A vital piece of information needed to distinguish between the two possibilities is a reliable measurement of proton concentration. In TFR proton concentration was measured by residual gas analysis after the termination of the discharge and values compatible with the mode conversion model were found. However, whether a similar fraction of protons also existed during the discharge is unclear. In ATC no gas analysis was carried out, but there are spectroscopic indications (SUCKEWER (45)) that the upper bound of the proton density was of the order of 58 of the deuteron density. The measurements are, however, not entirely conclusive. The definitive conclusions on the details of damping mechanism must therefore awalt more reliable information on the actual proton concentration.

In this paper we have also described.ICRF heating of ATC plasmas in the vicinity of the second harmonic of the deuteron cyclotron frequency. The measured heating is shown to represent an Increase in the thermal content of the bulk of the fon species. Heating is not limited to the tall of the ion distribution nor to the minority ion species. Comparable heating in both the perpendicular and parallel degrees of freedom is also observed. Furthermore, the heating occurs in the central core of the plasma. Maximum heating resulted in a $200-0 \mathrm{~V}$ increase in the ion temperature at an power level of $145 \mathrm{~kW}$. Heating efflelency based upon the Ion energy inerease and average rewer ranged from 10 to 408 when we take account of the finlte ion=energy confinement time. 


\section{ACKNOWLEDGEMENT}

The author would like to acknowledge many helpful discussions with Drs. T. H. Stix and F. W. Perkins. He would also like to thank Drs. H. Hsuan and S. Suckewer for providing bolometric and spectroscopic information. This work was supported by the Energy Research and Development Administration Contract EY-76-C-02-3073. 
REFERENCES

(1) Rothman, H. A., Sinclair, R. M., Brown, I. G., and Hosea, J. C., "Ion Cyclotron Heating in the Model C stellarator", Physics of Fluids, 12, 2211 (1969).

(2) Adam, J., and Samain, A., Report EUR-CEA-FC-579, (1971), ASSOCIATION EURATOM-CEA, Fontenay-aux-Roses.

(3) Ivanov, N. V., Kovan, I. A., and Los', E. V., "Excitation of the Spectrum of Natural Oscillations of the Plasma Pinch in the Tokamak To-1," Translation from Atomnaya Energiya, 32, 453, (1972). (4) Vdovin, V. L., Zinov'ev, O. A., Ivanov, A. A., Kozorovitskii, L. L., Parail, V. V., Rakhimbabaev, Ya. R., and Rusannv, V. D., "Excitation of Magnetosonic Resonance in the Tokamak Plasma", ZhETF Pis. Red. 14, 228, (1971).

(5) Hooke, W. M., and Hosea, J. C., "Wave Generation and Heating near the Ion Cyclotron Frequency in the ST Tokamak", Fifth European Conf. on Cont. Fusion and Plasma Physics, Grenoble, 1972, Vol. I, pl07, Commissalidt a L'Enenergie Atomique, Paris.

(6) Hosea, J. C., and Hooke, W. M., "Ion Cyclotron and Fast Hydromagnetic Wave Generation in the ST Tokamak", Phys. Rev. Lett., 31, 150, (1973).

(7) Adam, J., Chance, M., Eubank, H., Getty, W., Hinnov, E., Hooke, W., Hosea, J., Jobes, F., Perkins, F., Sinclair, R., Sperling, J., and Takahashi, H., "Wave Generation and Heating in the ST Tokamak at the Fundamental and Harmonic Ion Cyclotron Frequencies", in Proc. of the 5th Conf. on Plasma Physics and Cont. Nucl. Fusion Res., Tokyo, 1974, Int. Atomic Energy Agency, Vienna, 1975, Vol. II, p65: See Also: Takahashi, H., Report Matt-1140, 1975, Princeton Plasma Physics Lab. 
(8) Perkins, F. W., Chance, M., and Kindel, J. M., "Hydromagnetic Wave Heating of Tokamak Plasmas", Paper B-8, Third Int. Symposium on Toroidal Confinement, Garching, 1973, Max Planck Inst. f. Plasmaphysik, Munich, 1973.

(9) TFR-Group, "Excitation and Damping of the Fast Magnetosonic Wave in TFR near the Harmonic Cyclotron Frequency", 3rd Int. Meeting on Theo. and Exp. Aspects of Heating Toroidal Plasmas, Vol. I, p87, Commissariat A L'Energie Atomique, Grenoble, 1976. (10) TFR-Grcup, "Magnetosonic Wave Generation and Damping in the TFR Tokamak riear the Ion Cyclotron Frequencies", in Proc. of the 6th Conf. on Plasma Physics and Cont. Fusion Res., Berchtesgaden, 1976, Int. Atomic Energy Agency, Vienna, 1977, Vol. III, p39. (11) Vdovin, V. L., Shapotkovskii, N. V., and Rusanov, V. D., "Wave Generation and Heating of Ions at Ion Cyclotron Frequencies in Tokamak-Device TM-1-VCh", Proc. 3.rd Int. Meeting on Theoretical and Experimental Aspects of Heating Toroidal Plasmas, Vol. II, p349, Commissariat A L'Energie Atomique, Grenoble, 1976.

(12) Ivanov, N. V., Kovan, I. A., and Sokolov, Y. A., JETP Lett., $\underline{24}, 317(1976)$.

(13) Buzankin, V., Vershov, V. A., Ivanov, N. V., Kovan, I. A., Krupin, V. A., Popov, I. A., Semenov, I. B., and Sokolov, Y. U., "Experiments in Magnetoacoustic Heating of Plasma in Tokamak T-4", in Proc. of the 6 th Int. Conf. on Plasma Physics and Cont. Fusion Res., Berçhtesgaden, 1976, Int. Atomic Energy Agency, Vienna, 1977, Vol. III, p 61 .

(14) Greenough, N., Paoloni, F. J., and Takahashi, H., "Generation, Propayatiun and Damping of Fast Waves in Deuterium Plasmas in the ATC Tokamak", Bull. Am. Phys. Soc., 21, 1157 (1976). 
(15) Ivanov, N. V., Kovan, I. A., and Svishchev, V. S., "Particle and Energy Balance in Magnetosonic Heating of a Plasma in the TO-1 Tokamak", JETP Lett., 20, 39, (1974).

(16) Takahashi, H., Daughney, C. C., Ellis, Jr., R. A., Goldston, R. J., Hsuan, H., Nagashima, T., Paoloni, F. J., Sivo, A. J., and Suckewer, S., "RF Heating in ATC at the Ion Cyclotron Range of Frequencies (ICRF)", Bull. Am. Phys. Soc., 21, 1157 (1976). (17) Takahashi, H., Daughney, C. C., Ellis, Jr., R. A., Goldston, R. J., Hsuan, H., Nagashima, T., Paoloni, F. J., Sivo, A. J., and Suckewer, S., "Ion Heating in ATC Tokamak in the Ion Cyclotron Range of Frequencies", Phys. Rev. Lett., 39, 31, (1977). (18) Stix, T. H., "Fast Wave Heating of a Two-Component Plasma", Nuclear Fusion, 15, 737, 1975.

(19) Chance, M., Perkins, F. W., and Sperling, J., "Linear Absorption of Magnetosonic Normal Modes in a Tokamak Plasma", Bull. Am. Phys. Soc., 18, 1273 (1973). (20) Adam, J., and Jacquinot, J., "Eiqenmode Field strurture nf the Fast Magnetosonic Wave in a Tokamak and Loading Impedance of Coupling structure", Report EUR-CEA-FC-886, 1977, Association Euratom-CEA, Fontenay-Aux-Roses.

(21) Studier, P., Hsuan, H., Smith, R. R., and Takahashi, H., "On Heating and Heat Loss Mechanisms and Power Limit in the RF. Heating Experiments in the ATC Tokamak in the Ion Cyclotron Range of Frequencies (ICRF)", Bull. Am. Phys. Soc., 21, 1157 (1976). (22) Buchsbaum, S. J., "Resonance in a Plasma with Two Ion Species", Phys. of Fluids, 3,418 (1960).

(23) Buchsbaum, S. J., "Resonance in Multicomponent Plasma", Phys. Rev. Lett. , 5, 495, (1960). 
(24) Stix, T. H., The Theory of Plasma Waves, McGraw-Hill, New York, 1962, Sec. 3-8.

(25) Adam, J., Alvalez de Toledo, F., and Feltin, "Two-Ion

Collisional Absorption of Low Frequency Electromagnetic Waves in a Toroidal Hard-Core Configuration", Proc. of the 4 th European Conf. on Cont. Fusion and Plasma Physics, Rome, 1970, Comitato Nazionale per l'Energia Nuclear, Ufficio Edizioni Scientiche, Rome, 1970, pl05.

(26) Tarasenko, V. F., Ovchinnikov, S. S., Kalinichenko, S. S., Kurilko, P. I., Shvets, O. M., and Tolok, V. T., "High-Frequency Heating of a Plasma Under Conditions of Ion-Ion Hybrid Resonance", JETP Lett. 16,114 (1972).

(27) Burchenko, P. Ya., Volkov, E. D., Kramskoi, E. D., Longinov, A. V., Miroshnichenko, G. A., and Nizhnik, G. Ya., "High-Frequency Plasma Heating in a Stellarator", JETP Lett. 15, 174 (1972). (28) Klima, R., Longinov, A. V., and Stepanov, K. N., "High Frequency Heating of Plasmas with Two Ion Species", Nucl. Fusion, 15, 1157, (1975).

(29) Budden, K. G., Radio waves in the Ionosphere, Chapt. 21, Cambridge Univ. Press, Cambridge, 1961. (30) Stix, T. H. (1962), op.cit., Siec. 10-7,8,9.

(31). Swanson, D. G., "Mode Conversion and Tunneling at the Two-Ion Hybrid Resonance", Phys. Rev. Lett., 36, 316, (1976) .

(32) Jacquinot, J., McVey, B. D., and Scharer, J. E., "Mode Conversion of the Fast Magnetosonic Wave in a Deuterium-Hydrogen Tokamak Plasma", Phys. Rev. Lett., 39, 88 (1977). 
(33) Perkins, F. W., "Heating Tokamaks via the Ion-Cyclotron and Ion-Ion Hybrid Resonances", Princeton Plasma Physics Lab. Report, PPPL-1336, 1977.

(3.4) Yoshikawa, S., Rothman, M. A., and Sinclair, R. M.,

"Absorption of Ion Cyclotron Waves by one Component of a TwoIon Plasma", Phys. Rev. Lett., 15, 214, (1965).

(3.5) Stix, T. H., (1962), op.cit., p246.

(36) Bol, K., Ellis, R. A., Eubank, H., Furth, H. P., Jacobsen, R. A., Johnson, L. C., Mazzucato, E., Stodiek, W., and Tolnas, E. L., "Adiabatic Compression of the Tokamak Discharge", Phys. Rev. Lett., 29, 149.5. (1972).

(37) Stott, P. E., Daughney, C. C., and Ellis, Jr., R. A., "Control of Recycling and Impurities in the ATC Tokamak by Means of Gettered Surfaces", Nucl. Fusion, 15, 431 (1975). (38) Parsons, C. R., and Medley, S. S., "On the Interpretation of Charge-Exchange Ion Temperature Measurements in Tokamaks", Plasma Physics, 16, 267 (1974).

(39) Suckewer, S., and Hinnov, E., "Spectroscopic Measurements of Ion Temperatures in ATC with RF and Neutral Beam Heating", Bull. Am. Phys. Soc., 21, 1086 (1976).

(40) Stott, P., "Ion Energy Containment in Uncompressed Discharge in the ATC Tokamak", Plasma Physics, 18, 251 (1976).

(41) Stix, T.H., "Heating of Toroidal plasmas by Neutral Injection", Plasma Physics, 14, 367 (1972).

(42) Hildebrand, F. B., Advanced Calculus for Applications, Prentice-Ha11, Englewood Cliffs, N. J., 1962, Sec. 8.2. (43) Spitzer, L., Physics of Fully Tonized Gases, Interscicnce, New York, Eq. 5-31, pl35. 
(44) Spitzer, L., op.cit., Eq. 5-30, pl35.

(45) Sokolov, Y. A., private communication.

(46) Suckewer, S., private communication. 


\section{FIGURE CAPTIONS}

Fig. 1. Rf coupling structure used in the recent ATC experiments.

Fig. 2. (a) Cross-section of a Tokamak plasma. The resonant surface is roughly a cylindrical surface of a constant major radius. Thermal effects make the surface a region of a finite width shown shaded. Ions moving along a field line enters the region and receives a "kick" in their perpendicular energy. (b) An elementary physical picture of cyclotron harmonic acceleration. The wave field varies twice as fast as the ion Larmor motion. The ion is accelerated and then decelerated over one Larmor period, but its energy does not in general return to its original value due to inhomogenuity of the wave field.

Fig. 3. Oscillogram of magnetic probe signals showing toroidal cavity resonance and anti-resonance. The two probes are located near the top and bottom of the plasma.

Fig. 4. (a) Variation of the wave damping length normalized by the toroidal circumference as the toroidal field strength is varied. The abscissa is the major radius location of the second harmonic deuteron resonance layer. The region of the minimum damping length is asymmetric with respect to the nominal plasma center at $87 \mathrm{~cm}$. (b) Variation of bolometer signal which registers power loss through charge exchange and radiation. The net difference between discharges with and without rf pulse is plotted. The nominal extent of the plasma region is indicated. The outer bumper limiter is positioned at $107 \mathrm{~cm}$ '. 
Fig. 5. Oscillograms of output signals form an analog circuit which calculate equivalent series loading resistance of the antenna. Variation of the peak electron density for each case is also shown. The output from the circuit contain some instrumental effects and the ordinate of the oscillograms is only approximately proportional to the resistance. A qualitative trend of diminishing toroidal resonance can be seen.

Fig. 6. (a) Geometry of two-ion hybrid resonance/cut-off pair in a D-T plasma $\left(\mathrm{C}_{\mathrm{H}}=10 \%\right)$. The Tokamak center is to the right of the figure. In the shaded regions the fast wave is evanescent. (b) Variation of the cold plasma dielectric tensor elements along the meridian chord for the case shown in (a): (c) Variation of the square of the perpendicular refractive index.

Fig. 7. Polarization of the fast wave electric field near two-ion hybrid resonance. Rotation of the E-field vector is also indicated (B-field is upward). Rapid variation of the polarization over a small distance between the hybrid and proton cyclotron resonance is notable. A cold plasma with $\mathrm{n}_{\mathrm{eo}}=2.5 \times 10^{19} / \mathrm{m}^{3}, \mathrm{C}_{\mathrm{H}}=3 \%$ and $n_{n}=12$ are assumed.

Fig. 8. Slab geometry for boundary value problem. The static B-field is in the positive $z$-direction (upward) and $x$-axes point to the high field side. Parabolic electron density and ion temperature profiles and a Tokamak-like B-field variation are assumed.

Fig. 9. (a) Variation of $E_{X}$-component of the wave electric field across the slab for three different values of $B$ : One for which the proton cyclotron resonance falls at the plasma center 
$(90 \mathrm{~cm})$ and two others for which the resonance is displaced by $6 \mathrm{~cm}$ on either side of the center. The slab half-width is $17 \mathrm{~cm}$. The hybrid resonance has only small effects on $E_{X}$, but they are asymmetric with respect to the center. (b) Variation of $E_{+}-f i e l d$ for the same three cases. The hybrid resonance has strong effects on $\mathrm{E}_{+} \cdot$ Note the appearance of a very steep gradient between the hybrid and cyclotron resonance layers.

Fig. 10. Normalized damping length as a function of the location of the proton cyclotron resonance layer. The damping is due to proton cyclotron damping (minority heating) only. The curve labeled $w / O$ HBR is without influence of the hybrid resonance. The hybrid resonance enhances the damping somewhat and accentuate the asymmetry.

Fig. 11. Dependence of the normalized damping length onproton concentration.

Fig. 12. Significance of BUDDEN's parameters. A plot of the perpendicular refractive index squared against the normalized coordinate is shown in the vicinity of the hybrid resonance. $\beta^{2} / n^{2}$ is the asymptotic value of $n_{\perp}^{2}$, and $-n^{2} / \beta$ is the thickness of the evanescent layer between the resonance and cut-off.

Fig. 13. (a) Dependence of BUDDEN's parameters on proton concentration. The location of the cyclotron layer is fixed at the plasma center $(90 \mathrm{~cm})$ and the concentration is varied. $\left(n_{\text {eo }}=2.5 \times 10^{19} / \mathrm{m}^{3}, n_{n}=12\right)$. Fall-off of $\beta$ and $n$ at high values of $\mathrm{C}_{\mathrm{H}}$ is due to lower densities at the hybrid layer. (b) Variation of BUDDEN's parameters as the cyclotron resonance moves across the plasma. Asymmetry of variation with respect to the center is again noticeable. 
Fig. 14. Simplified boundary value calculations based upon BUDDEN's tunnelling factor. Plane waves bounce back and forth between two reflecting boundaries and progress also in z-direction. Each time they go through the hybrid layer they suffer absorption of a part of their energy.

Fig. 15. Cyclotron and cyclotron harmonic resonances of various ions. The ordinate is the ratio of the atomic weight to the electronic charge number of ions. The abscissa is the "locations" of the resonances in terms of the normalized major radius, normalized B-field or normalized frequency. The condition for the $n$-th harmonic resonance is given by $y=n / x$. The range-bars and a circle with ATC, PLT and TFTR indicate example of the use: of this chart (see text).

Fig. 16. Perpendicular charge exchange neutral spectra with and without an rf pulse. The inset shows an oscillograph of threechannel charje exchange analyzer signals (inverted) (5 ms/div): the analyzer settings are from top to bottom, 1.1252 .25 and $4.5 \mathrm{keV}$.

Fig. 17. (a) Radial profiles of ion temperature determined from various temperature measurements: charge exchange, ovII, $\Delta$ CV, CIV: Open symbols without and solid symbols with rf heating (b) Charge exchange temperature (solid lines) and neutron temperature ( $\bullet$ with and $O$ without rf heating) in discharges compressed at $30 \mathrm{~ms}$.

Fig. 18. Charge exchange signal relaxation process after termination of the rf pulse. $\bar{s}$ is the $C x$ signal amplitude normalized by its value at the pulse end. Theory is shown by solid curves and experiments by : (a) time evolution at $\mathrm{E}=2.7 \mathrm{keV}$ (b) Dccay time to half-value ayainst energy. 
Fig. 19. (a) Evolution of perpendicular temperature: 0 with rf heating, o without rf heating: (b) Incremental temperature rise attributable to rf heating: The abscissa is time-averaged rf power divided by total number of ions in the device (in units of $10^{19}$ ). 


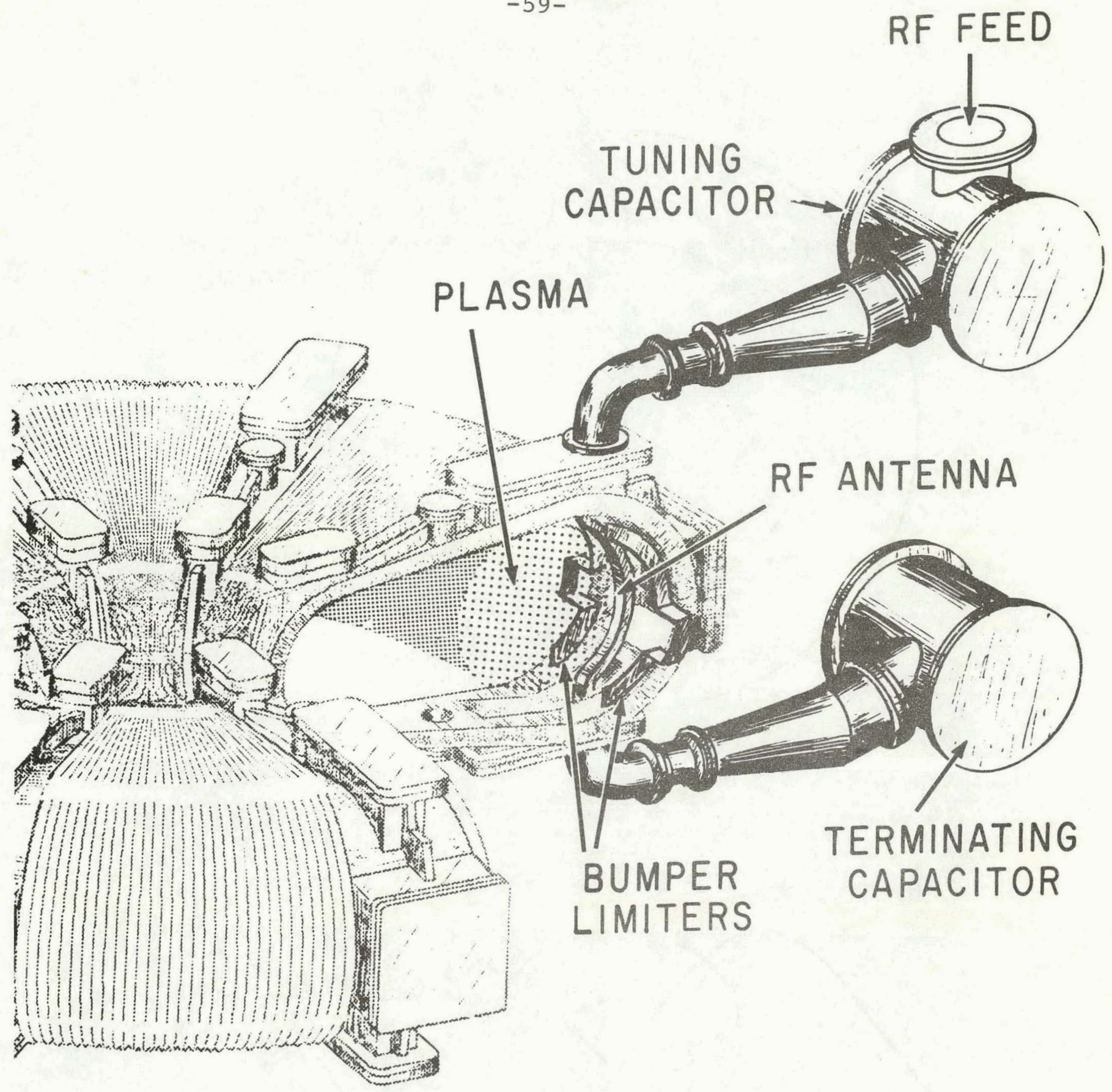

Fig. 1. 766223 


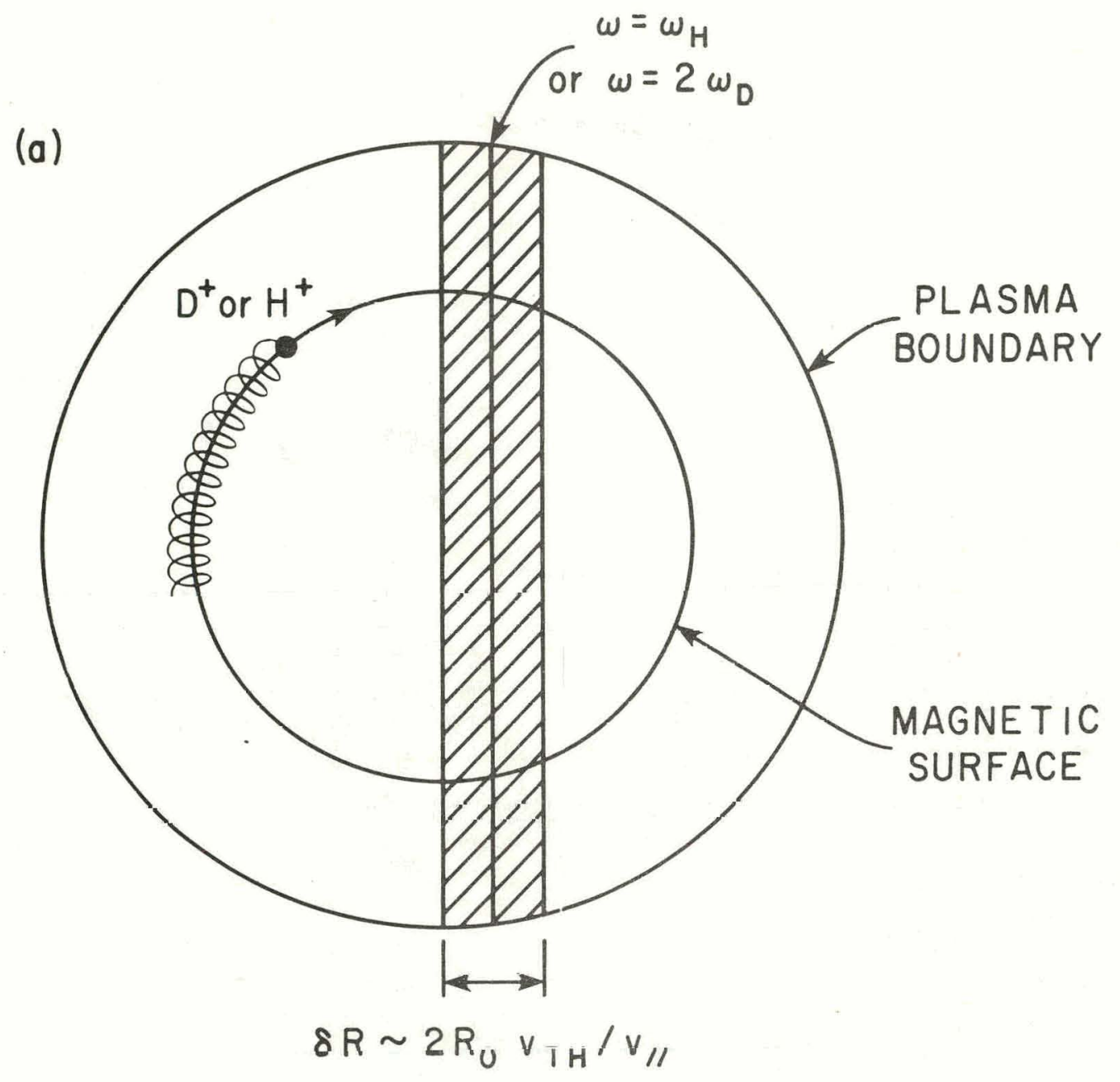

(b)

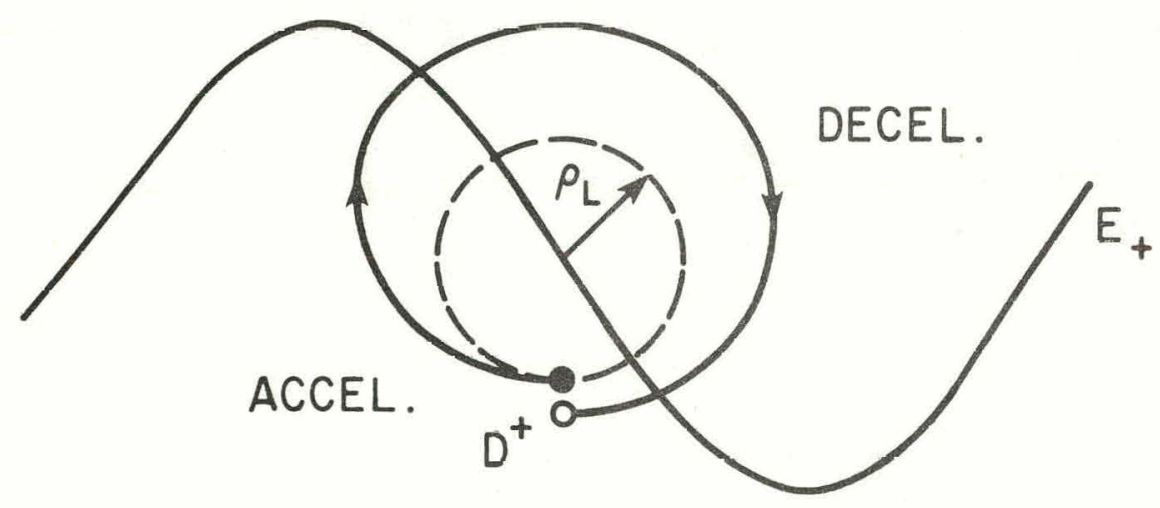

$$
\begin{gathered}
E_{+}, k_{\perp} \neq 0 \\
P \sim 1 / 2\left(k_{\perp} \rho_{L}\right)^{2}\left|E_{+}^{2}\right|
\end{gathered}
$$

Fig. 2. 773471 
$\downarrow \sqrt{ }$ RESONANCE

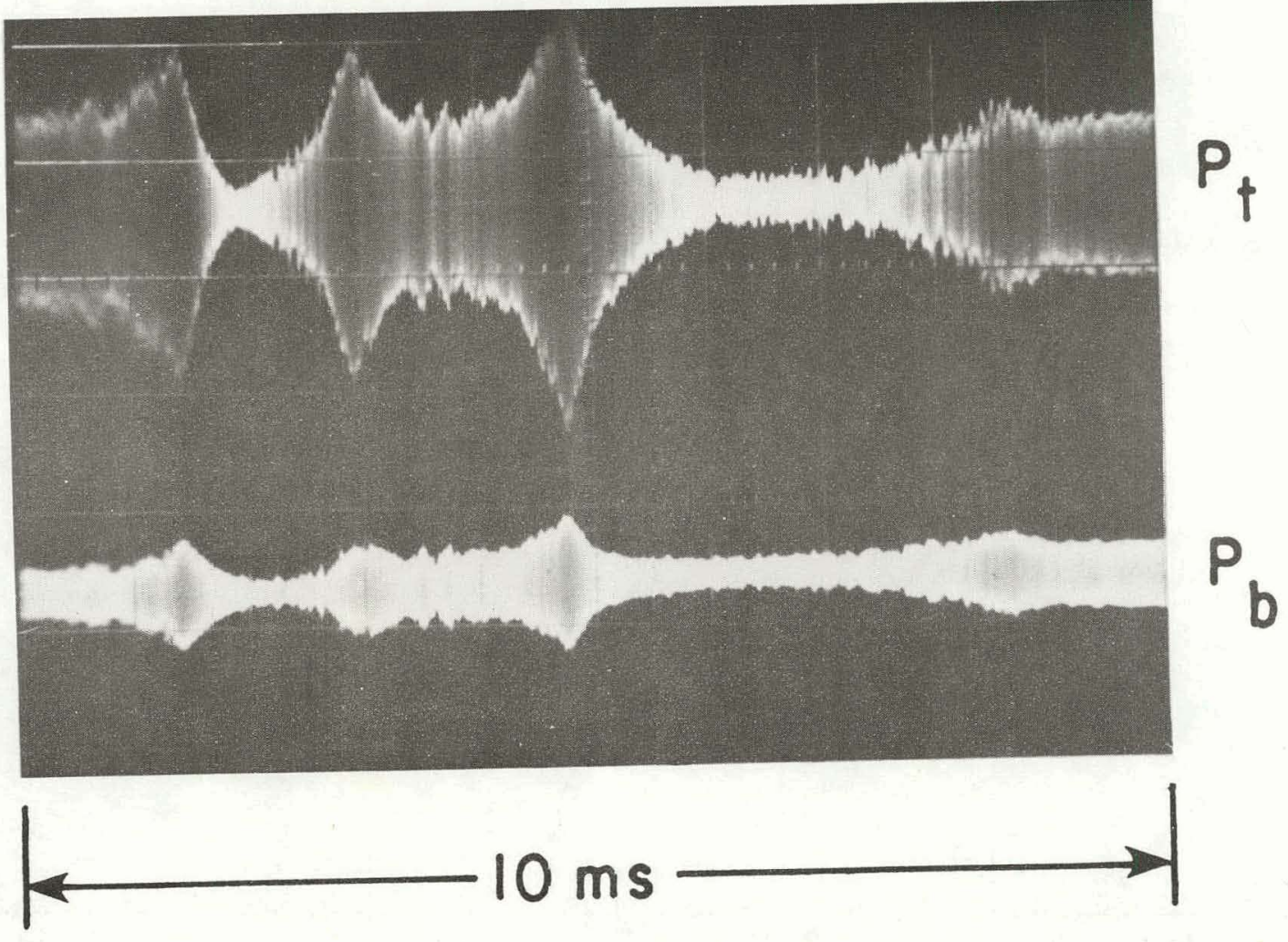



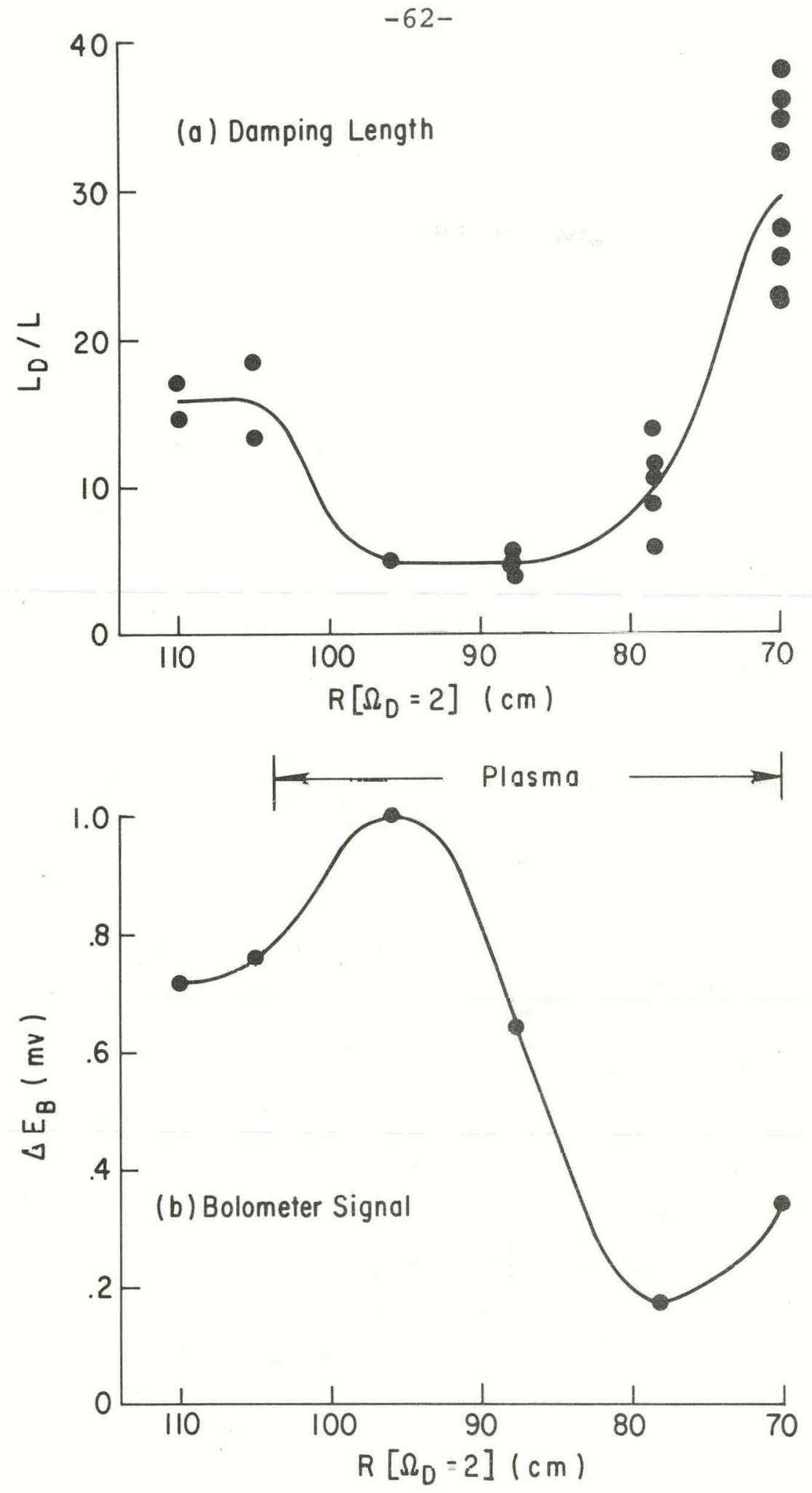

Fig. 4. 773466 
$-63-$
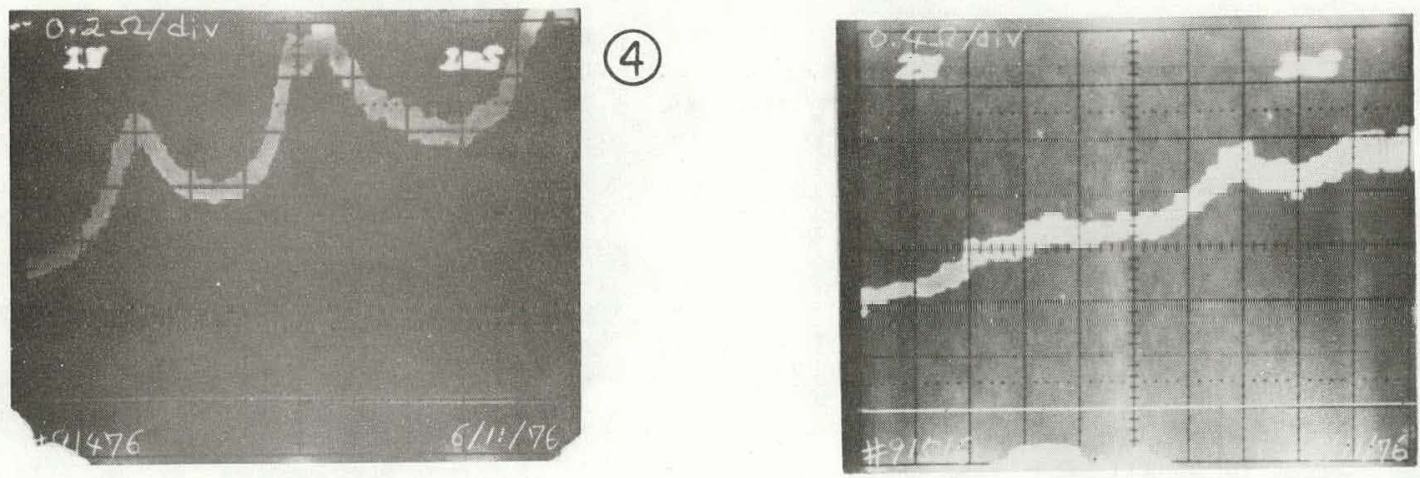

(5)
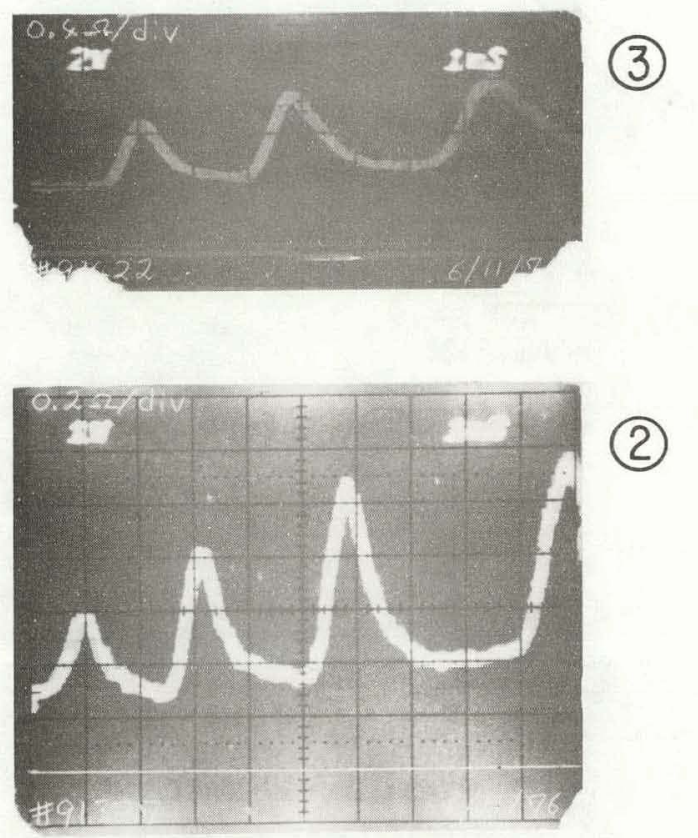

(2)
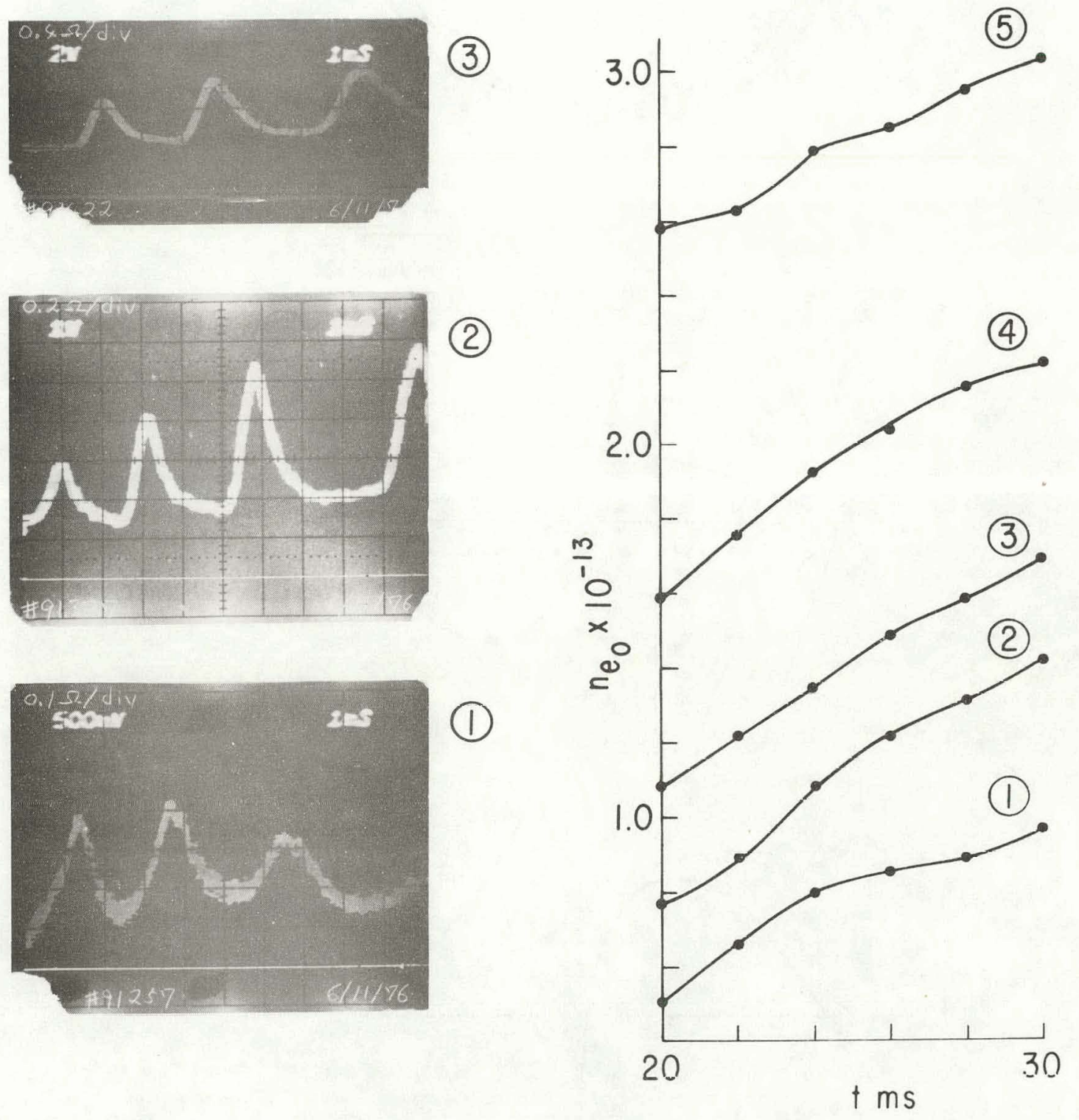

Fig. 5. 766046 


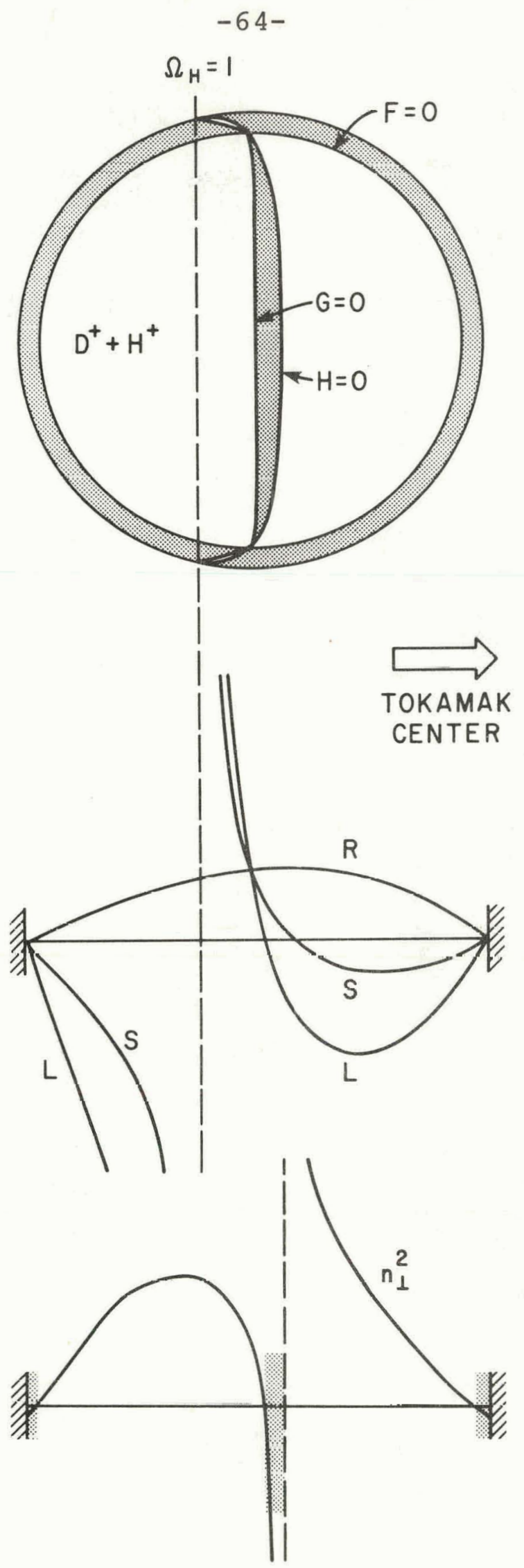

Fig. 6. 773495 


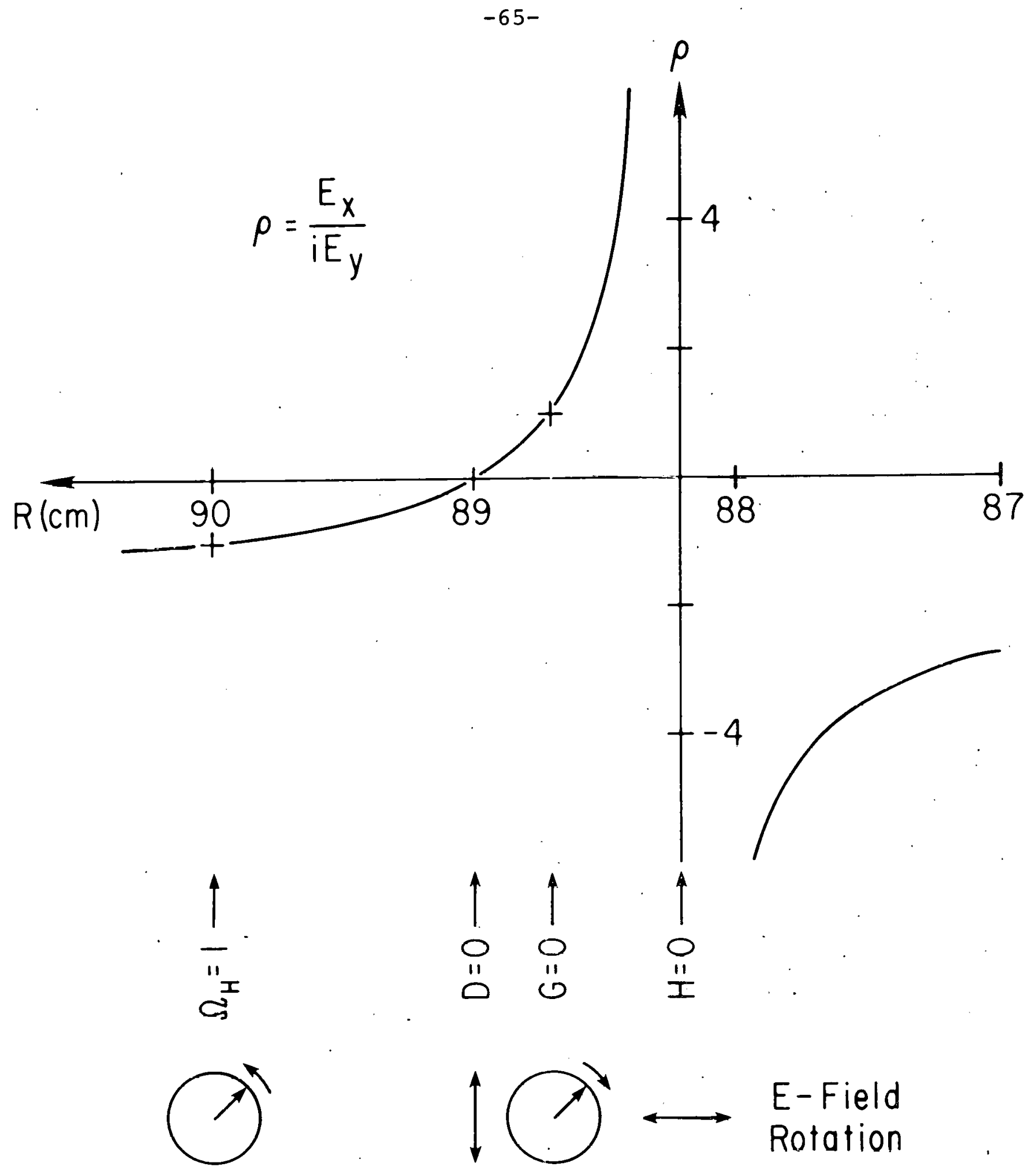

Fiy. 7. 773493 


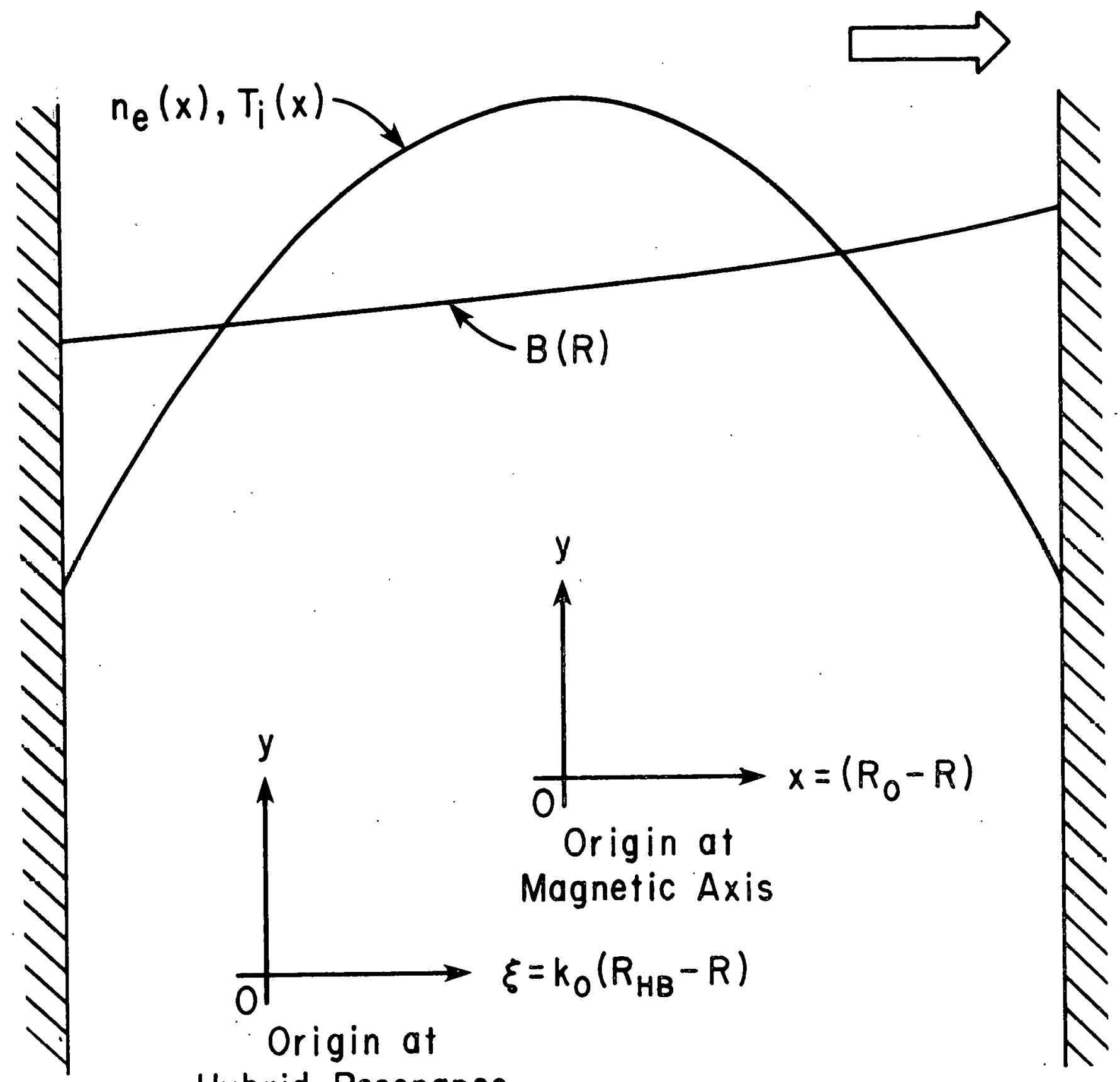

Hybrid Resonance

Fig. 8. $\quad 773477$ 

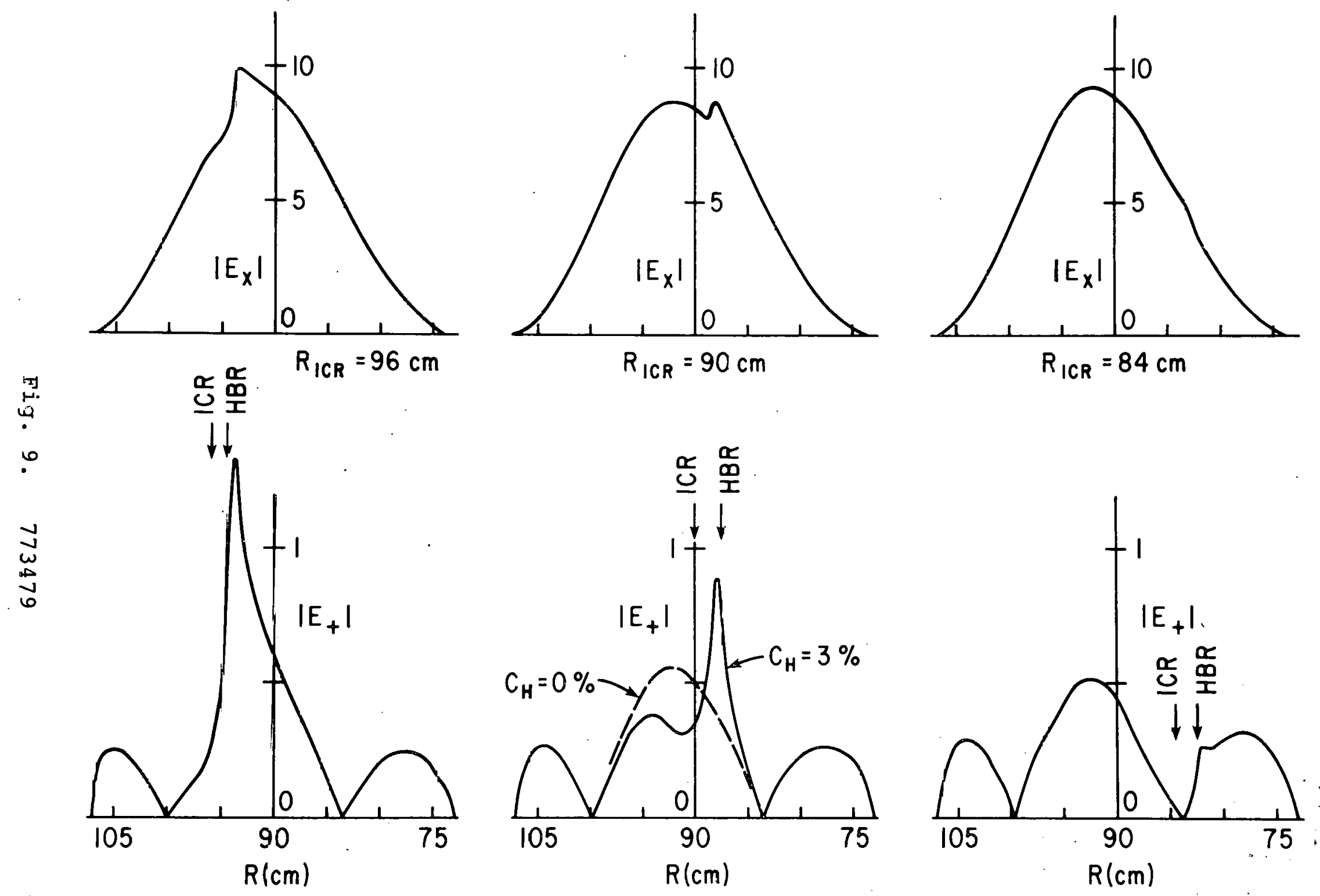


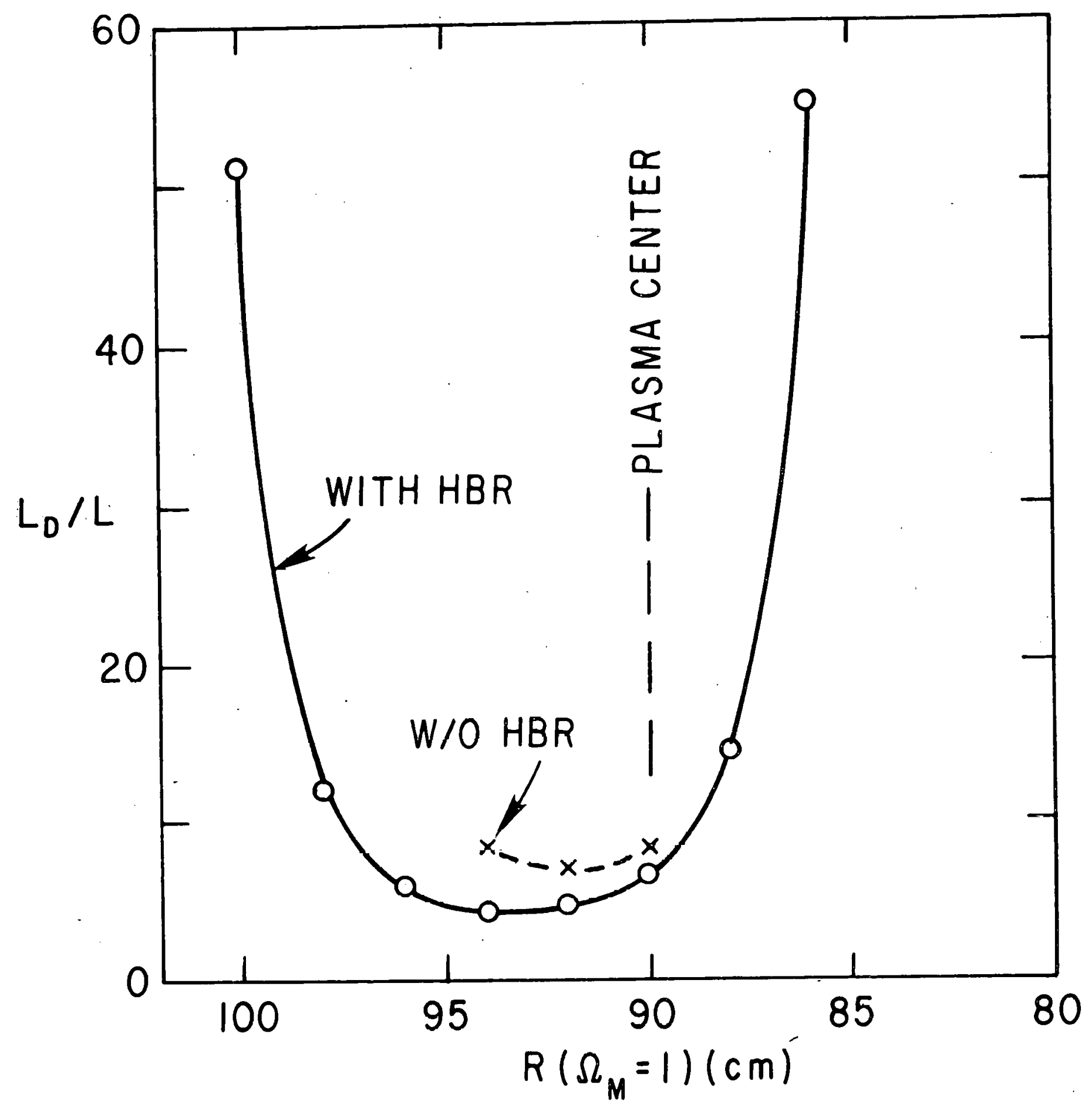

Fig. 10. $\quad 773470$ 


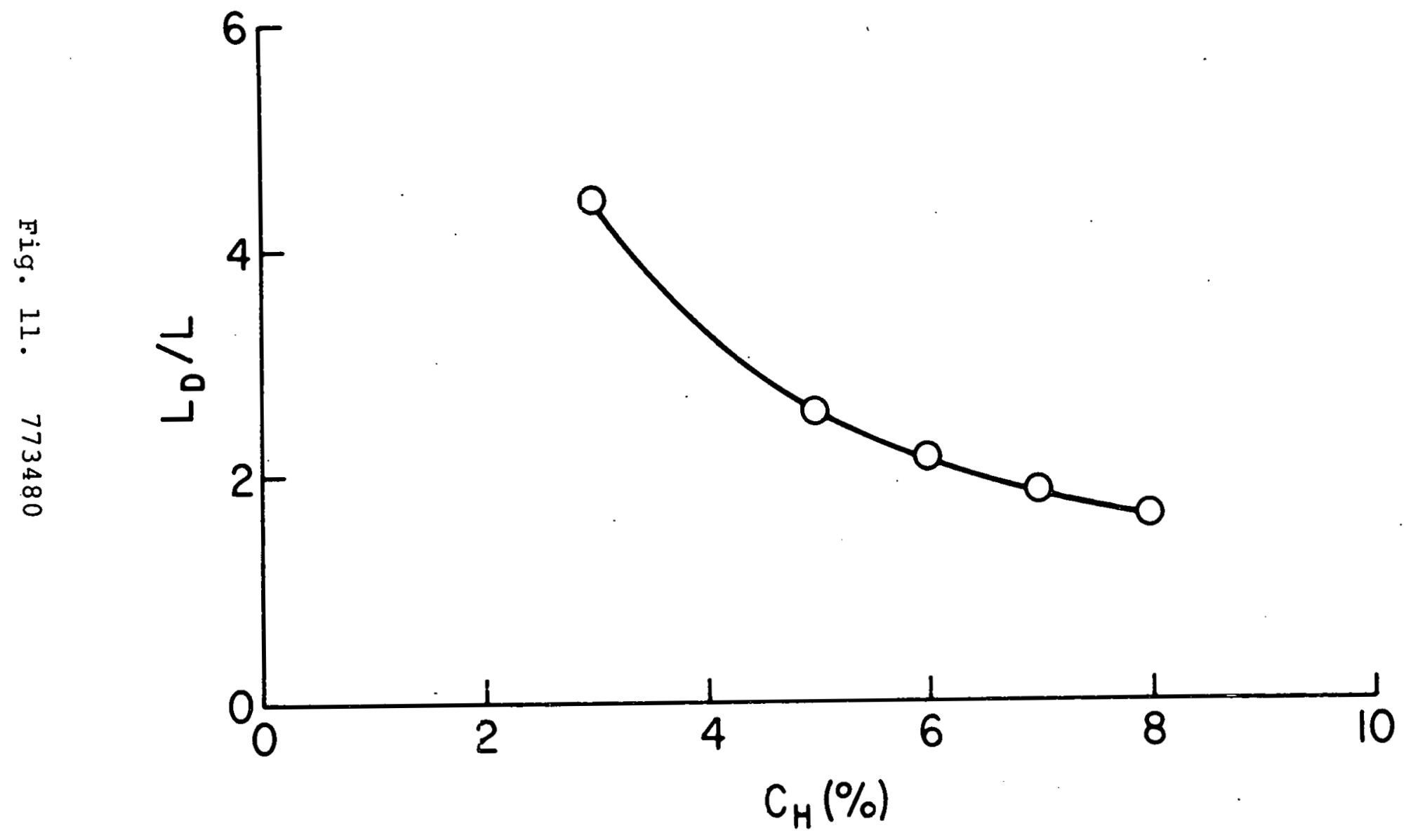




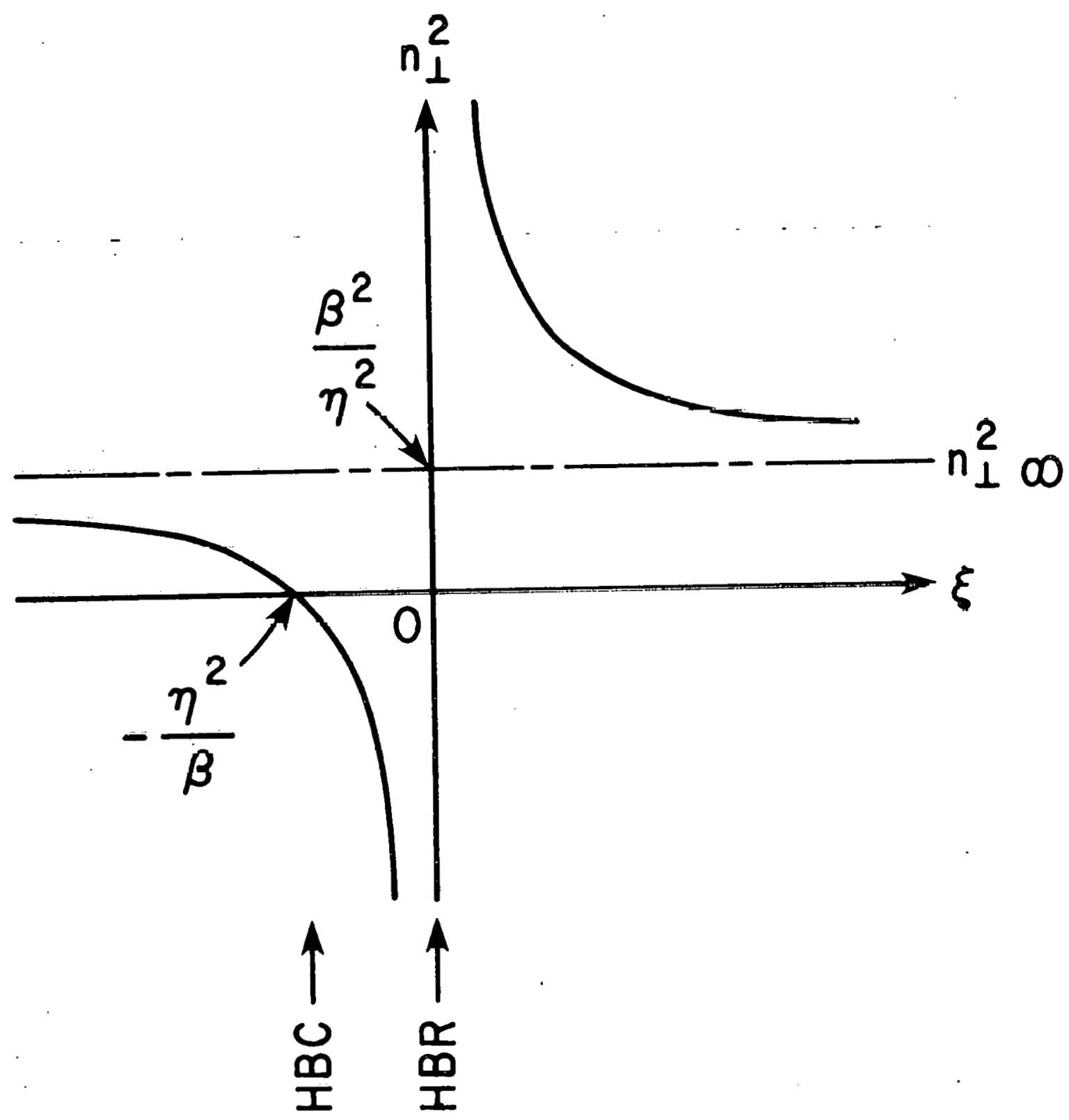

Fig. $12 \quad 773481$ 

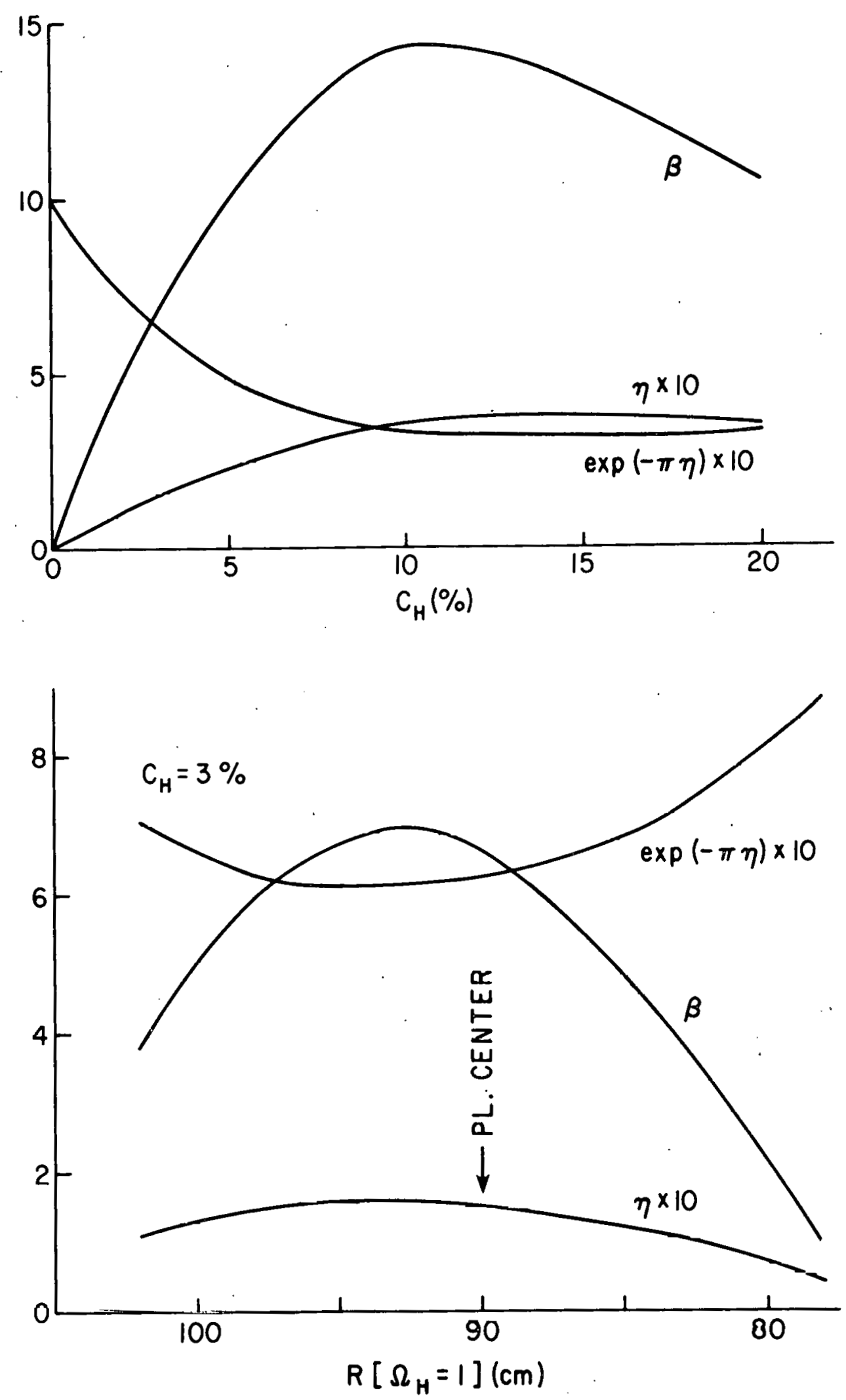

Fig. 13. 773478 


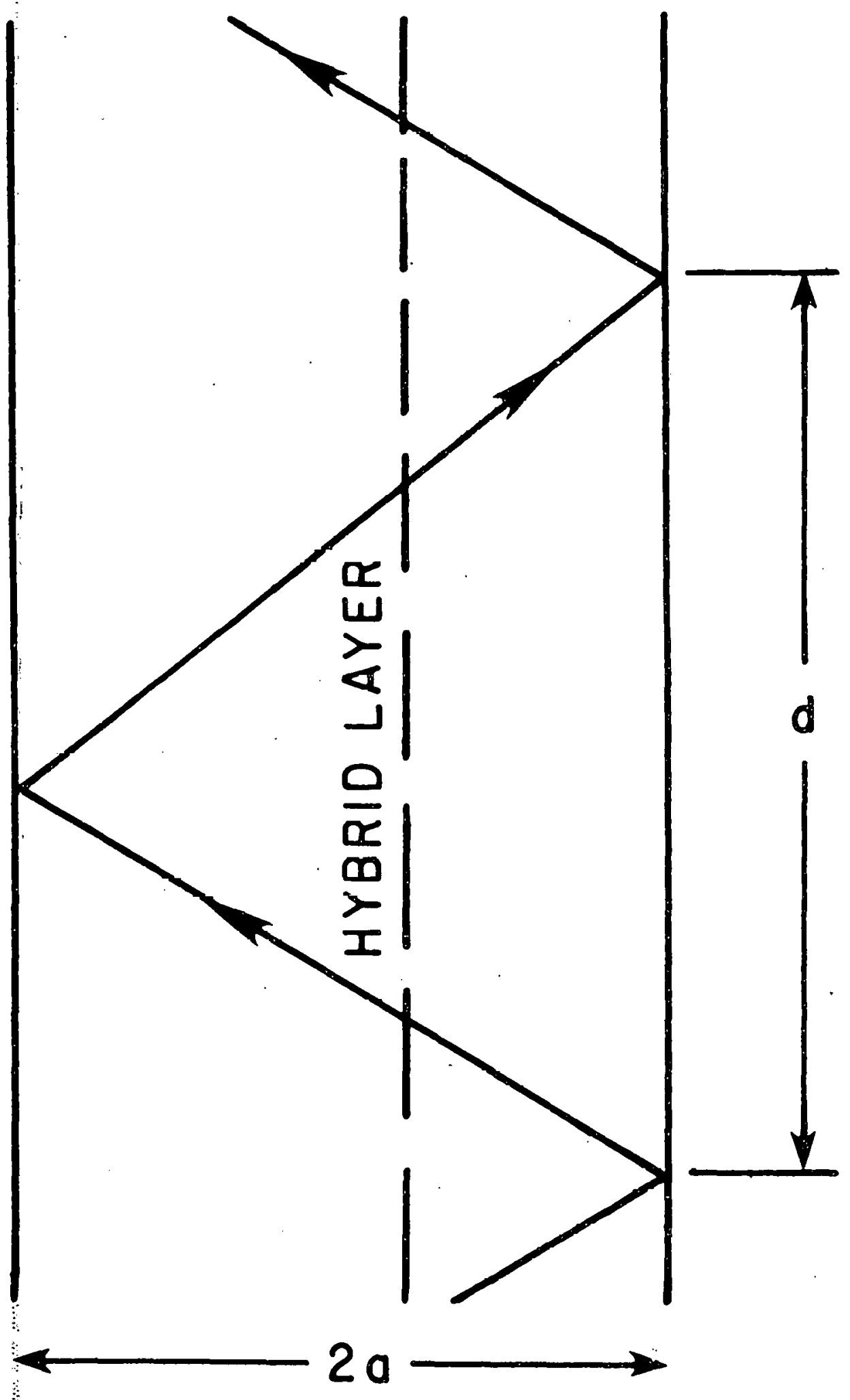

Fig. 14. 773494 


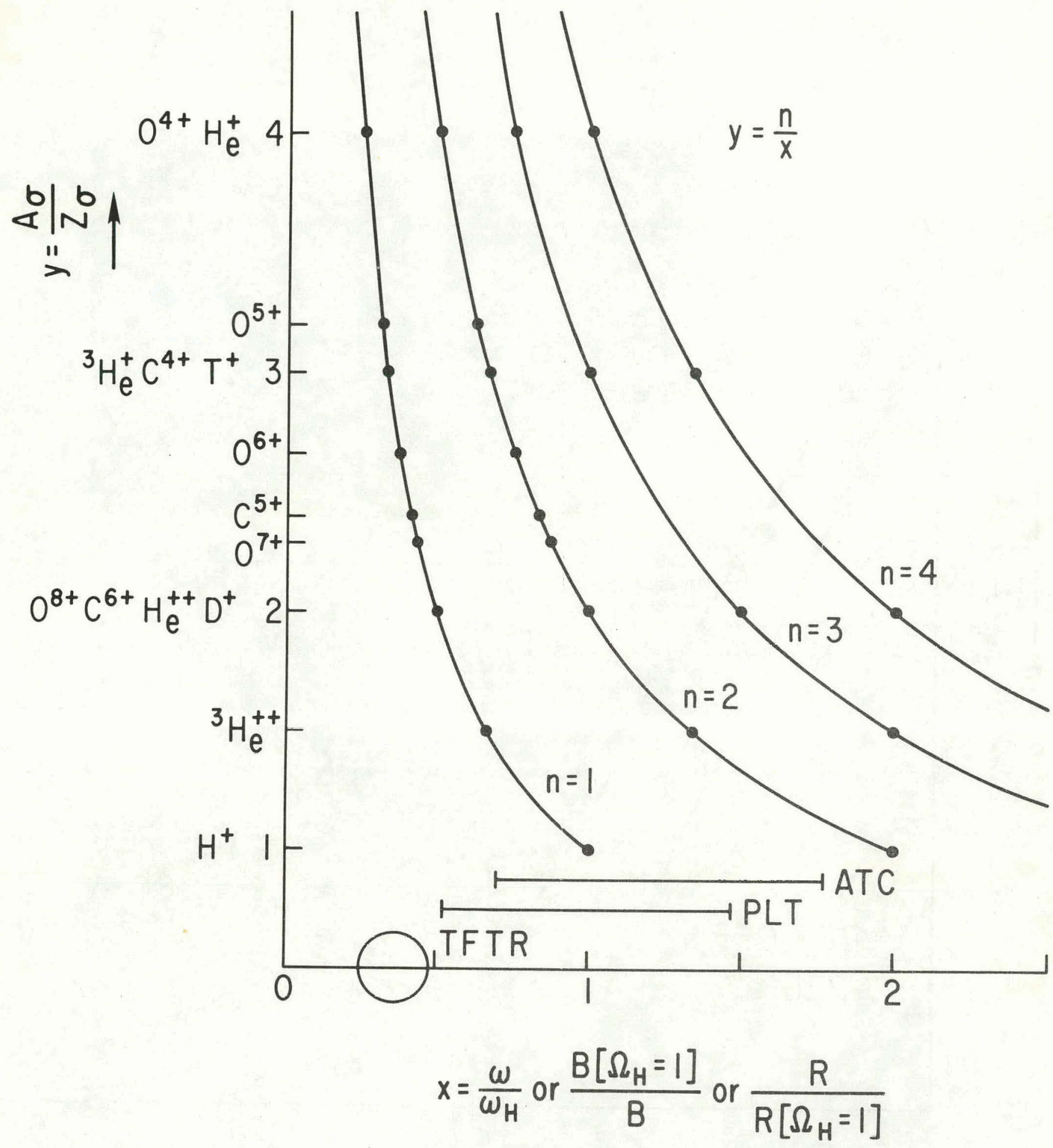

Fig. 15. 773492 


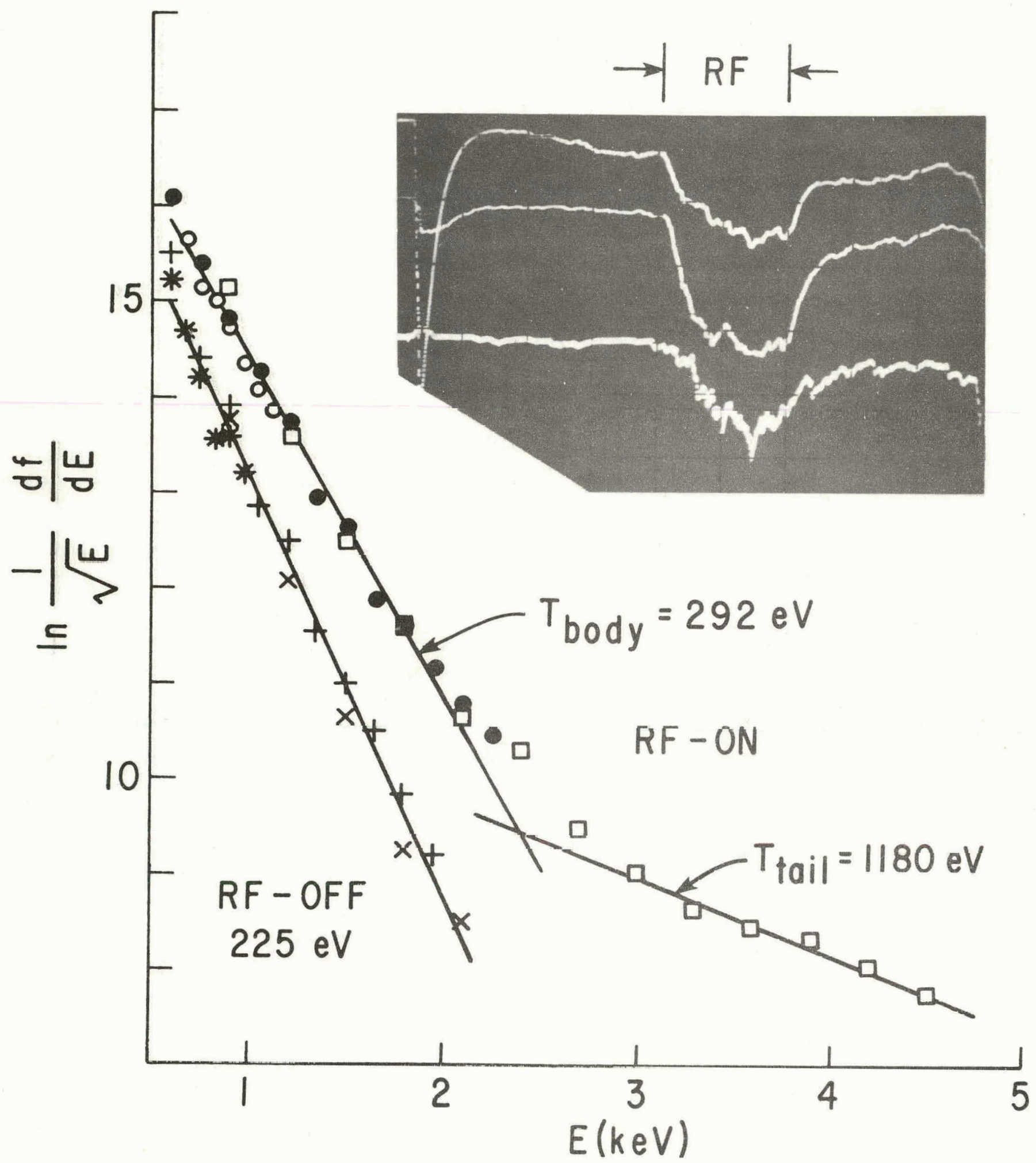

Fig. 16. 773284 


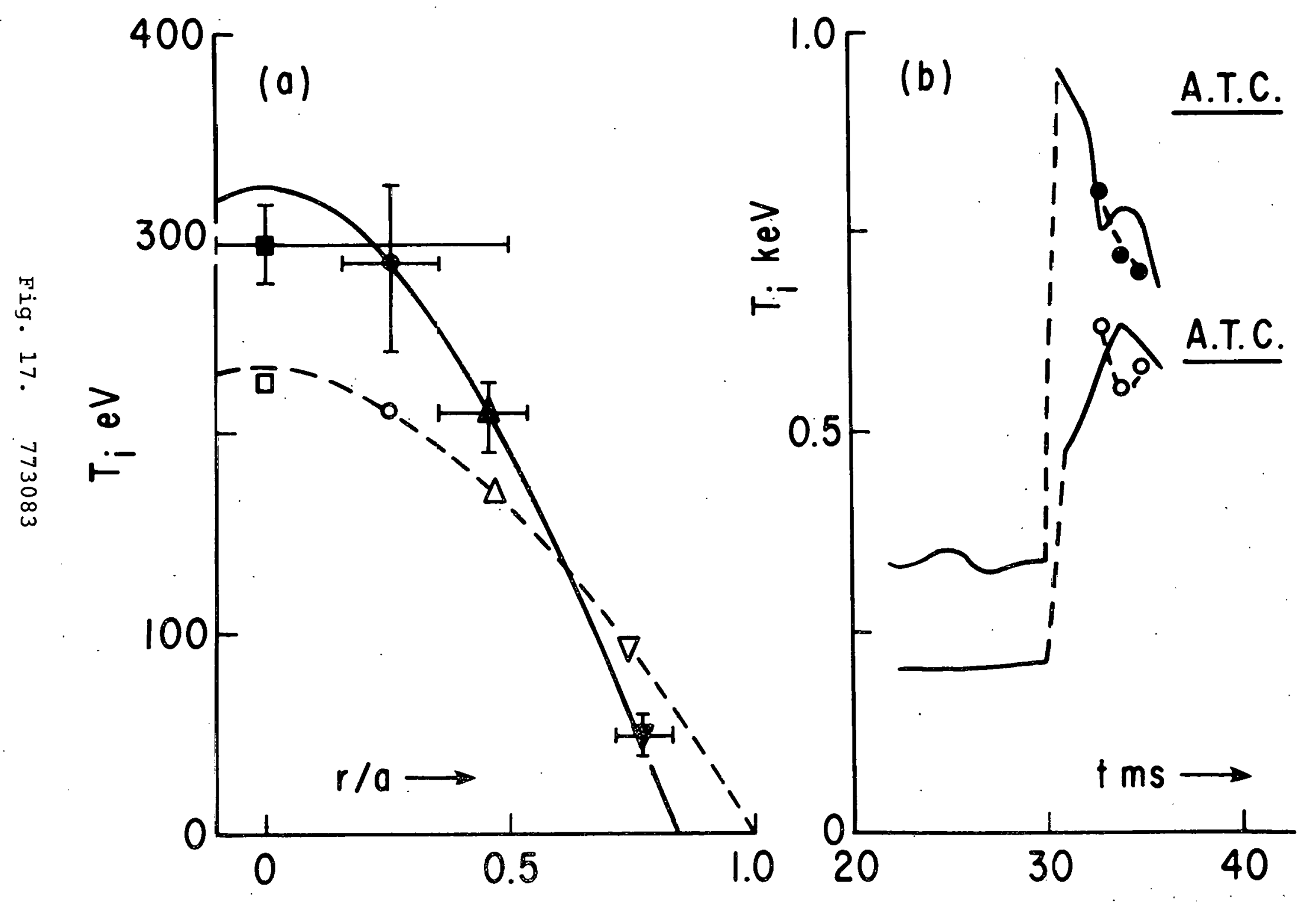



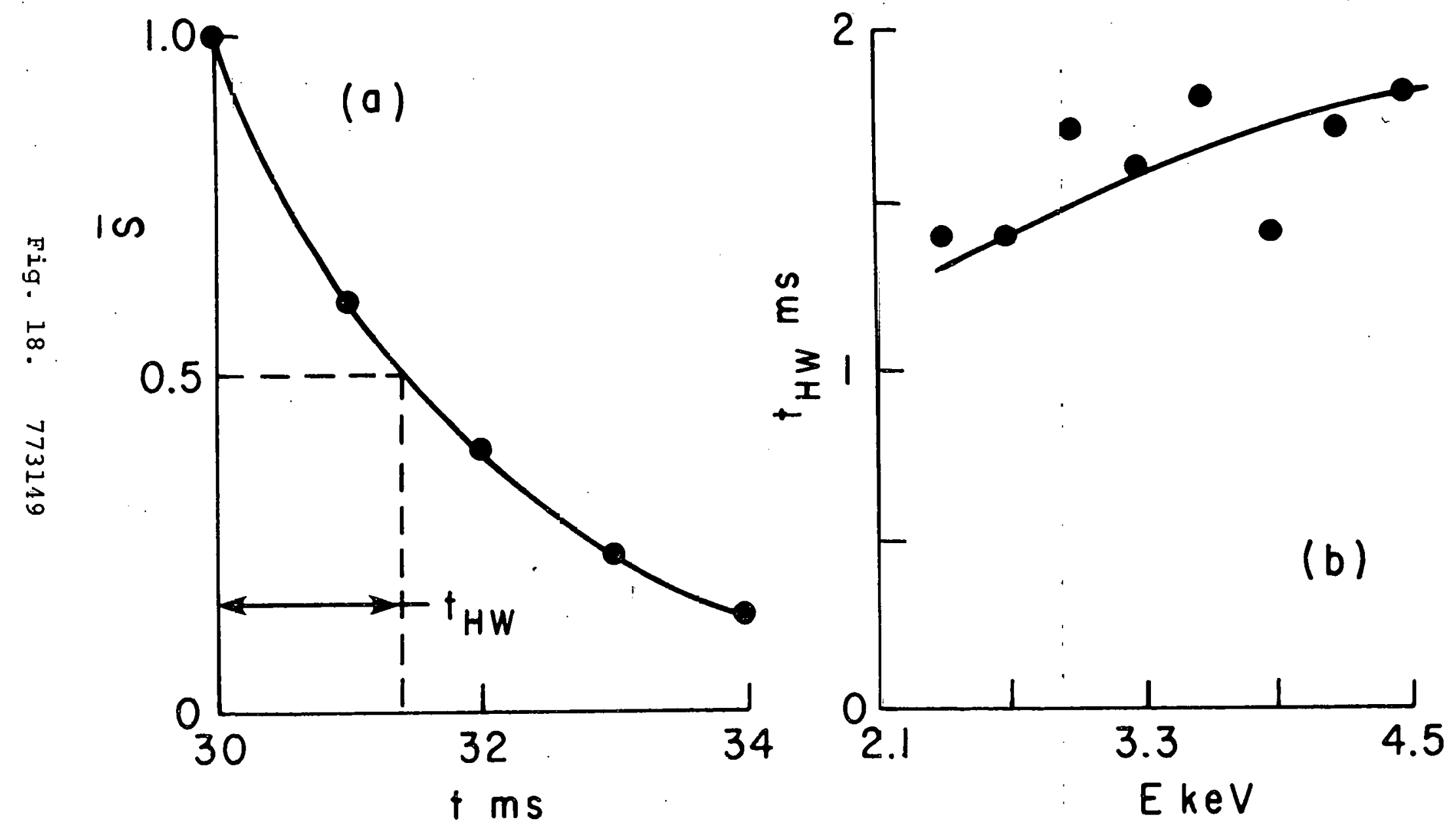

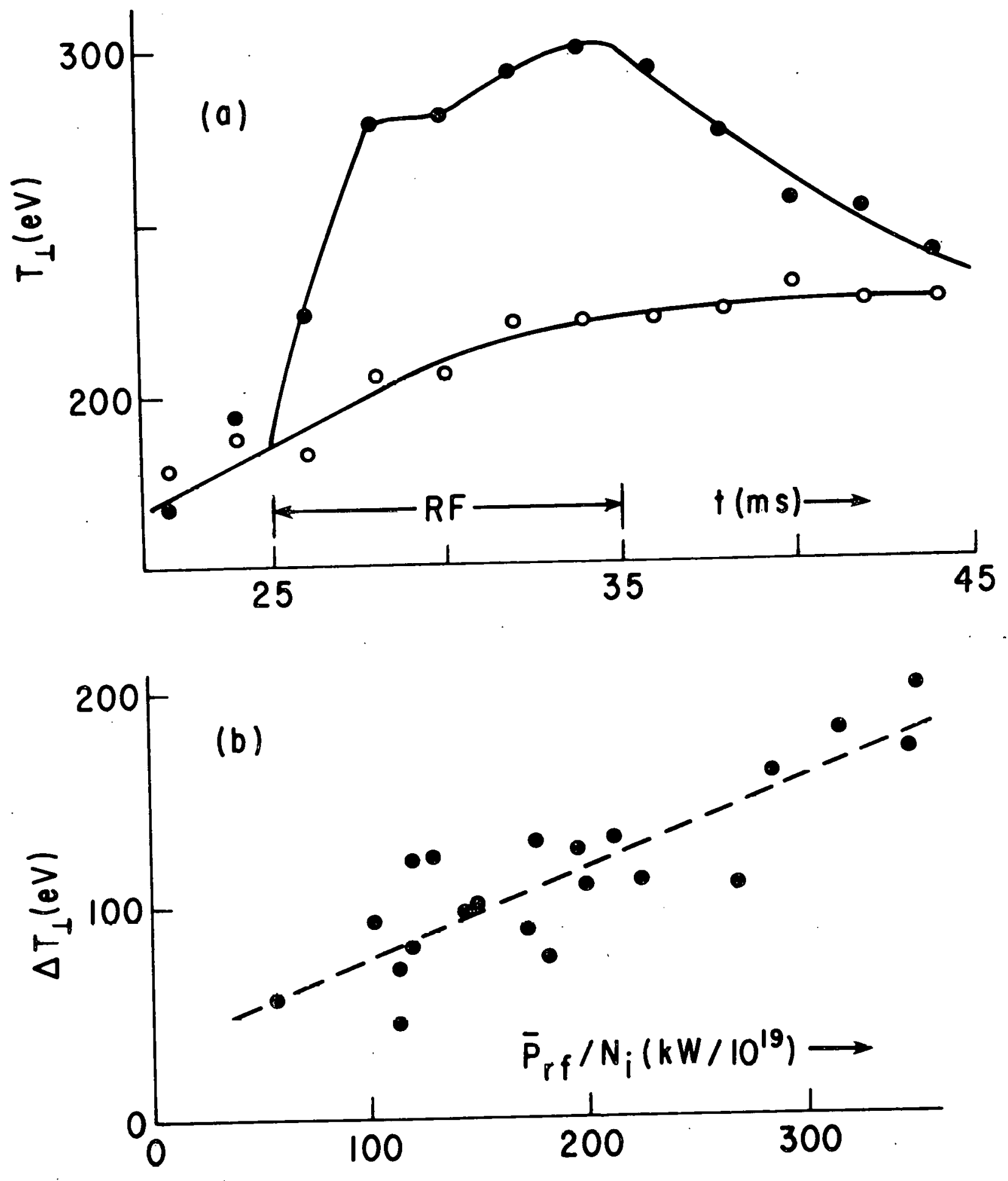

Fig. 19.. 773283 